hep-th/0408192

SLAC-PUB-10670, SU-ITP-04/32

UCB-PTH-04/22, UMD-PP-05/015

LBNL-54768I

\title{
In the Realm of the Geometric Transitions
}

\author{
Stephon Alexander ${ }^{1,2}$, Katrin Becker ${ }^{3}$, Melanie Becker ${ }^{4}$ \\ Keshav Dasgupta ${ }^{1}$, Anke Knauf ${ }^{4,5}$, Radu Tatar ${ }^{6}$ \\ ${ }^{1}$ Department of Physics, Stanford University, Stanford CA 94305 \\ ${ }^{2}$ SLAC, Stanford University, Stanford CA 94309 \\ stephon, keshav@itp.stanford.edu \\ ${ }^{3}$ Department of Physics, University of Utah, Salt Lake City, UT 84112 \\ katrin@physics.utah.edu \\ ${ }^{4}$ Department of Physics, University of Maryland, College Park, MD 20742 \\ melanieb@physics.umd.edu, anke@umd.edu \\ ${ }^{5}$ II. Institut für Theoretische Physik, Universität Hamburg \\ Luruper Chaussee 149, 22761 Hamburg, Germany \\ ${ }^{6}$ Theoretical Physics Group, LBL Berkeley, CA 94720 \\ rtatar@socrates.Berkeley.EDU
}

\begin{abstract}
We complete the duality cycle by constructing the geometric transition duals in the type IIB, type I and heterotic theories. We show that in the type IIB theory the background on the closed string side is a Kähler deformed conifold, as expected, even though the mirror type IIA backgrounds are non-Kähler (both before and after the transition). On the other hand, the Type I and heterotic backgrounds are non-Kähler. Therefore, on the heterotic side these backgrounds give rise to new torsional manifolds that have not been studied before. We show the consistency of these backgrounds by verifying the torsional equation.
\end{abstract}

Work Supported in part by the Department of Energy Contract DE-AC02-76SF00515

Stanford Linear Accelerator Center, Stanford University, Stanford, CA 94309 


\section{Contents}

1. Introduction . . . . . . . . . . . . . . . . . . . . . . . . . . . . . . 1

2. Supergravity Analysis in Type IIA . . . . . . . . . . . . . . . . . . . . . 7

2.1. Background before geometric transition . . . . . . . . . . . . . . . . . 8

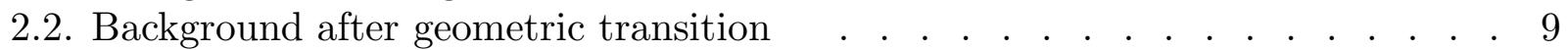

3. Supergravity Analysis in Type IIB . . . . . . . . . . . . . . . . . . . . . 10

3.1. Background before geometric transition . . . . . . . . . . . . . . . . . 10

3.2. Background after geometric transition . . . . . . . . . . . . . . . . . 11

4. Supergravity Analysis in Type I . . . . . . . . . . . . . . . . . . . . . . 23

4.1. Background before geometric transition . . . . . . . . . . . . . . . . . 26

4.2. Background after geometric transition . . . . . . . . . . . . . . . . . 41

5. Supergravity Analysis in Heterotic Theory . . . . . . . . . . . . . . . . . 45

5.1. Background before geometric transition . . . . . . . . . . . . . . . . . 46

5.2. Background after geometric transition . . . . . . . . . . . . . . . . . 48

6. Field Theory and Geometric Transition in Type I and Heterotic String . . . . . 50

6.1. Geometric transition . . . . . . . . . . . . . . . . . . . . 52

\section{Introduction}

One of the most active areas of research during the last year has been directed towards deepening the links between string theory and realistic supersymmetric gauge theories. Four years ago three different approaches linked non conformal $\mathcal{N}=1$ supersymmetric gauge theories with D-branes wrapped on cycles of Calabi-Yau manifolds and flux compactifications of ten-dimensional string theories [1] [2] [3]. The approach of [1] dealt with the conifold supergravity solution describing a cascade of Seiberg dualities from the UV to the IR. The IR theory is a deformed conifold, where the conifold singularity was replaced by an $S^{3}$ cycle.

The approach of [2] was based on a type IIA duality [4] between open topological strings on Lagrangian submanifolds and closed topological strings on a resolved conifold. By identifying IR gauge invariant quantities (gluino condensate) with volumes in the resolved conifold, a powerful duality between wrapped D6 branes and flux compactifications was developed. The type IIA duality was then extended to many examples of mirror type IIB duals [5] [6] [6] [8] [10] [11]. In the type IIB theory the duality relates the IR dynamics on D5 branes wrapped on resolution $P^{1}$ cycles and fluxes on $S^{3}$ in a deformed conifold.

In [3] the supergravity solution of an NS5 brane wrapped on a two cycle of a nontrivial background was analyzed. This approach mainly discussed the open string side of the type IIB duality, where supersymmetry was broken to $\mathcal{N}=1$ by performing a 
twist. These type IIB developments were stimulated by the fact that there is an already well-known and accepted form for the flux superpotential [12]

$$
W_{I I B}=\int\left(H_{R R}+\tau H_{N S}\right) \wedge \Omega
$$

where $H_{R R}$ is the RR flux, $H_{N S}$ is the NS flux, $\tau$ is the axion-dilaton and $\Omega$ is the holomorphic three-form. For most cases that were considered, the 3-cycles of the manifold were $P^{1}$ fibres over elliptic curves and this allowed a direct connection to Seiberg-Witten curves [5] and matrix models [10] [11].

The duality on the type IIA side is harder to study because Lagrangian submanifolds that can be wrapped by D6 branes are not easy to obtain. One important ingredient in the discussion of [2] was the fact that the duality required a closed string side with $d \Omega \neq 0$, i.e. a non-complex, non-Kähler manifold. As the mathematical apparatus to describe these manifolds has not yet been developed their construction turns out to be difficult as well. The first step in this direction was taken in [13] where the results of [14] were used to describe half-flat manifolds as examples of backgrounds with $d \Omega \neq 0$.

One very explicit example of type IIA duality was worked out in [15] (other type II models with torsion appeared in [16] [17] [18][19]). Starting with a conformal Calabi-Yau solution on the type IIB side corresponding to D5 branes wrapped on the resolution $P^{1}$ cycle of the resolved conifold [20] the mirror type IIA picture was obtained performing three T-dualities using the Strominger, Yau and Zaslow (SYZ) technique [21] 1 . Since the SYZ technique works properly when the base of the manifold is much bigger than the $T^{3}$ fiber, the limit of large complex structure was taken2. By identifying three angular directions, the type IIA dual with D6 branes wrapped on 3-cycles inside a non-Kähler deformation of the deformed conifold 3 was obtained.

1 Brane configurations obtained by one T-duality from configurations with D5 branes wrapped on $P^{1}$ cycles have been discussed in [22] 23] [24] [25] 26].

2 There is a subtlety though. The technique of [21] is valid in the limit where the base is large, whereas geometric transitions occur in exactly the opposite limit. Therefore using three $\mathrm{T}-$ dualities, we should not expect to get the right metric. In fact, this is exactly what was shown in [15] as the metric obtained by naive $\mathrm{T}$-dualities was missing some crucial components. The correct metric was only extracted after some non-trivial coordinate transformations were performed [15].

3 Examples with D6 branes wrapped on generalized calibrated submanifolds inside non-Kähler spaces have been recently discussed in [27]. 
Furthermore, the non-Kähler geometry with wrapped D6 branes was connected to another non-Kähler geometry with fluxes [15]. This was done by lifting the type IIA configuration with D6 branes to a manifold with torsion and $G_{2}$-structure by performing a flop in this seven-dimensional manifold. This allowed us to replace D6 branes by RR two-form fluxes and the torsion classes of the deformed geometry with the torsion classes of the resolved geometry. The final result was an $\mathcal{N}=1$ compactification with $\mathrm{RR}$ fluxes on a non-Kähler manifold with $d J \neq 0$ and $d \Omega \neq 0$ with a superpotential 13

$$
W_{I I A}=\int(J+i B) \wedge d \Omega \text {. }
$$

The goal of the present paper is to connect the closed type IIB theory to the closed type IIA theory, something that was not done in [15]. We shall show that the result is a complex Kähler manifold with a closed three-form $\Omega$ and a superpotential given by (1.1). By taking the same limit of large complex structure, we perform three $\mathrm{T}$-dualities to go to a type IIB geometry where the limits taken in [15] are naturally reconstructed. In other words, the limits of large complex structure between the type IIB geometries with D5 branes and with fluxes are naturally identified. This is a very important check of our duality cycle and confirms our approach.

Having completed the cycle of geometric transitions in the type IIA and type IIB theories we will extend this cycle to the type I and heterotic strings. To do so we use Sen's idea [28 of going to an orientifold limit of the type IIB theory and from there to the type I theory by performing two T-dualities. More precisely, we first need to build a metric which is invariant under the orientifold action by eliminating terms of the type IIB metric that are not invariant under the orientifold operation. Our metric is valid only at the orientifold point, in the perturbative regime. When non-perturbative effects become relevant, the orientifold plane splits into $(p, q)$ seven branes and when these, together with the initial D7 branes, are moved to infinity, the usual type IIB metric should be recovered 1 . This assumption will be crucial later when we determine the metric at the orientifold point.

When the type I string is compactified on a non-Kähler manifolda the superpotential (1.1) is mapped into [29]

$$
W_{I}=\int\left(H_{R R}+i d J\right) \wedge \Omega
$$

4 It remains an open problem how to describe the intermediate region, when there is a nontrivial axion-dilaton and the metric is to be determined from the Seiberg-Witten curve.

5 In principle it could be also non-complex, as in type IIA, but the Donaldson-Uhlenbeck-Yau equation for the gauge bundle requires the manifold to be complex. 
and the extra contribution from the D7 branes in type IIB is mapped to the holomorphic Chern-Simons term for the $\mathrm{SO}(32)$ gauge field.

The natural final step is to connect this theory to the heterotic string compactified on a non-Kähler manifold. This theory has been studied in great detail over the last few years [30] 31] [32] 33] [34] [35]. The manifold obtained on the heterotic side is a non-Kähler manifold and the fluxes will appear as torsion [36]. In this theory the moduli stabilization is achieved via the new superpotential proposed in [29] (see also [34])

$$
W_{\text {het }}=\int(H+i d J) \wedge \Omega
$$

where $H$ is the heterotic three-form satisfying $d H=\operatorname{tr} R \wedge R-\frac{1}{30} \operatorname{tr} F \wedge F$.

An important aspect of our discussion is that we provide geometric transition duals for both the type I and heterotic theories. Whether this implies transitions for $(0,2)$ theories is not yet clear6. In order to check the existence of such a transition one should identify the "sources" for the holomorphic superpotentials as topological strings. Some results for the $(0,2)$ topological strings have appeared in [40], but more progress is needed in order to clarify this issue.

Another rather important aspect of our U-dual type I/heterotic string configurations might be their relevance for string cosmology, along the lines of [41] for the type IIB theory. Indeed, within all of the corners of the M-theory moduli space, the heterotic string is the most studied one and developed towards making contact with the standard model of particle physics. In light of these developments an important goal of string theory is to find consistent de Sitter vacua and a realization of inflation in the context of the heterotic theory by identifying the inflaton field as a natural extension of the standard model.

In order to stabilize all the moduli fields of the heterotic string, compactification on non-Kähler manifolds have to be considered. When the non-Kähler manifold is compact, the radius can be shown to be stabilized at tree level by balancing the fluxes with the non-Kählerity of the internal space [29]. In the simplest case only the dilaton remains to be stabilized by including non-perturbative effects. On the other hand, for a Calabi-Yau compactification, both the radius and the dilaton have to be fixed by non-perturbative effects. This has been recently discussed in 42].

6 There are results related to definitions of mirror symmetry for such theories [37] [38] [39], but a geometric transition has not yet been proposed. 
Interesting solutions of strongly coupled heterotic string theory in de Sitter space have recently been obtained in [43]. Here it was shown that the balancing of two non-perturbative effects, coming from open membrane instantons stretching between the boundaries of heterotic M-theory and gluino condensation on the hidden boundary, leads to meta-stable de Sitter vacua. It was possible to predict the masses of charged scalar matter fields, the scale of supersymmetry breaking and the gravitino mass in a phenomenologically interesting $\mathrm{TeV}$ scale.

Cosmological relevant models might be constructed by considering compactifications of the heterotic on non-Kähler manifolds. The above observations are rather encouraging because the manifolds that we will construct here are all non-Kähler. It remains to be seen if our concrete Klebanov-Strassler type IIB solutions are relevant for cosmology. This will be left for future work.

Our analysis in this paper will follow the road outlined in the figure below:

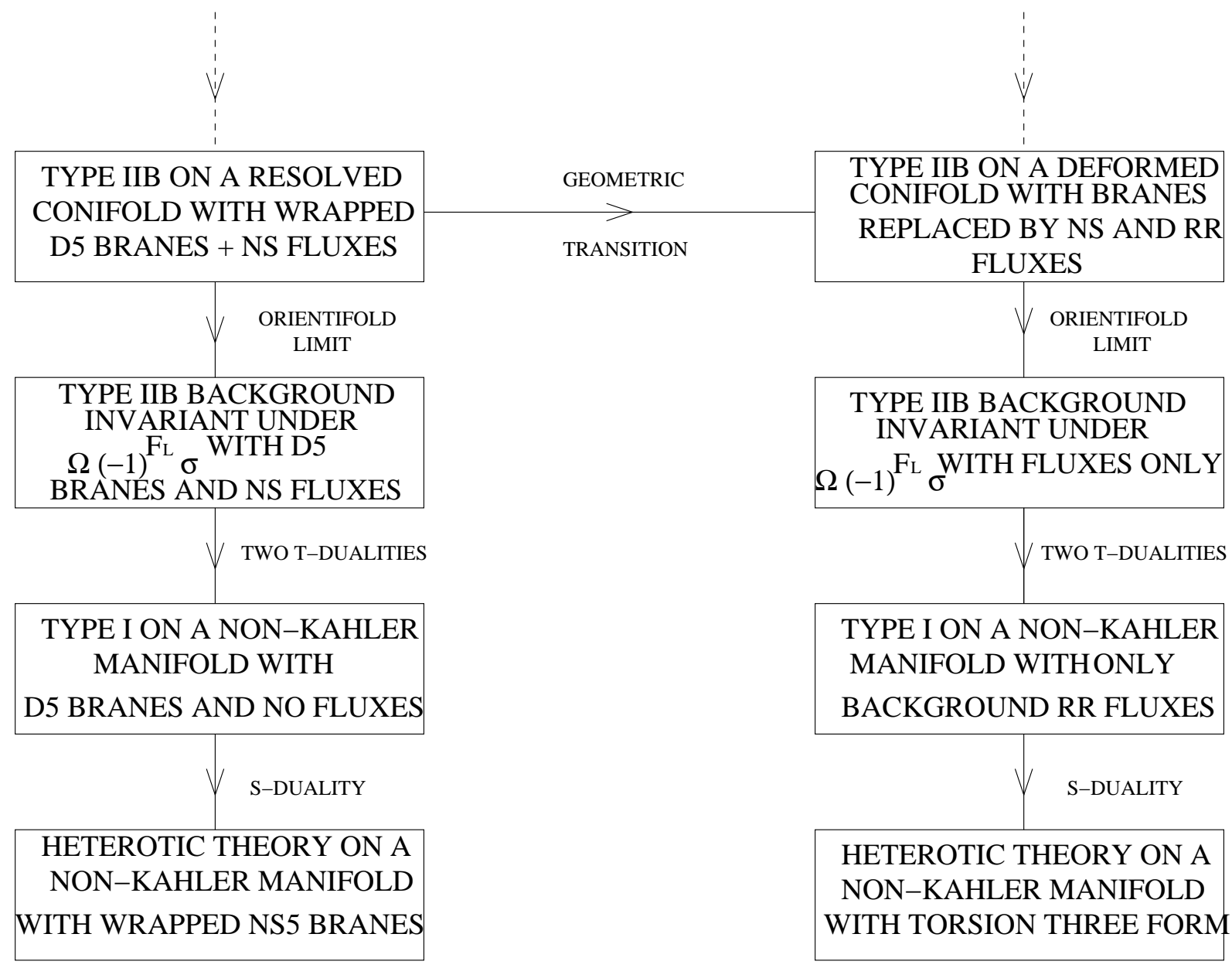

The boxes represent the duality chains and we will follow the arrows. The dotted arrows are the earlier duality chains encountered in [15]. Here we show that there is a new geometric 
transition for the type IIB theory. The type I heterotic duals will be obtained by going to the orientifold corner of the type IIB compactification. It is tempting to argue that there is a possible geometric transition between the two sides, although we will not make such a claim herein.

Our starting point will be the type IIA background which we had discussed in great detail in [15]. We will summarize the complete background both before and after the geometric transition in section 2. These backgrounds will be used to go to the mirror picture. We shall see that the mirror manifolds in the type IIB theory both before and after the geometric transition has taken place are complex Kähler manifolds, even though the corresponding type IIA manifolds were non-Kähler! This has been predicted earlier in [1], [3], [2], and will be derived here from a SYZ type analysis [21]. Section 3 contains the full analysis. Section. 3.1 gives a short summary of the type IIB background before the geometric transition. This was studied earlier in [20]. New results are presented starting in section 3.2, where we derive the Klebanov-Strassler kind background.

Having obtained the type IIB background, we then go to the orientifold limit. This is achieved by uplifting the type IIB backgrounds to F-theory. F-theory naturally incorporates $D 7$ branes and $O 7$ planes. This will be explained at the beginning of section 4 . We derive the metric of the type IIB theory at the orientifold point and use this to reach the type I theory. The type I metric is derived in section 4.1. Section 4.2 is devoted to the analysis after the geometric transition. The type I theory will show two different backgrounds: one in which we have $D 5$ branes wrapped on two-cycles of a non-Kähler manifold, and another one in which we have RR three-forms on another non-Kähler manifold. However, it is not clear to us whether this implies that if we shrink the two cycle with wrapped $D 5$ branes then we get another manifold with no branes but RR fluxes. More work needs to be done to reach any concrete conclusion. These details appear in sec. 4 .

In section 5. we go to the heterotic background. In section 5.1 we derive the nonKähler manifold with NS5 branes wrapped on a two cycle. In section 5.2 we derive another non-Kähler manifold with no branes but three-form torsion. The manifolds that we get in the heterotic theory are new examples of non-compact complex non-Kähler manifolds. So far most of the examples presented in the literature were compact complex non-Kähler

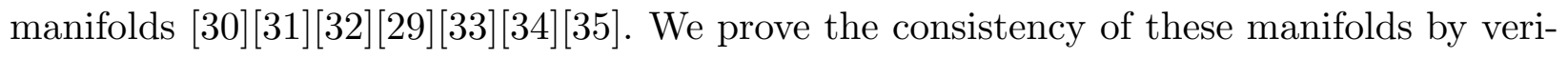
fying that they satisfy the torsional constraint [44] [36].

Section 6 is devoted to studying some generic properties of the field theory, superpotential and geometric transitions for the type I and heterotic theories.

Note added: While we were typing up our results, a paper appeared on the archive 445] that has some overlap with our work. 


\section{Supergravity Analysis in Type IIA}

In [15] an exact supergravity description of the type IIA geometric transition of [2] appeared where the backgrounds both before and after the transition were presented. In this section we will summarize the main results. The type IIA background was obtained from the type IIB background involving D5 branes wrapping a two cycle of the resolved conifold.

The metric of $D 5$ branes wrapped on a two cycle of the resolved conifold is

$$
d s^{2}=\left(d z+\Delta_{1} \cot \theta_{1} d x+\Delta_{2} \cot \theta_{2} d y\right)^{2}+\left|d z_{1}\right|^{2}+\left|d z_{2}\right|^{2}
$$

Here we have replaced the metric of the two spheres of the resolved conifold with two tori with complex structures $\tau_{1}$ and $\tau_{2}$. The complex one forms $d z_{i}, i=1,2$, are defined as

$$
d z_{1}=d x-\tau_{1} d \theta_{1}, \quad d z_{2}=d y-\tau_{2} d \theta_{2}
$$

For the case that was studied in [15], the complex structures $\tau_{i}$ can be chosen to be either integrable or non-integrable, and they are in general given by

$$
\tau_{1}=f_{1}+\frac{i}{2} \sqrt{\gamma \sqrt{h}}, \quad \tau_{2}=f_{2}+\frac{i}{2} \sqrt{\left(\gamma+4 a^{2}\right) \sqrt{h}}
$$

Here, $f_{i}$ are not yet specified and $\gamma, a$ are defined in [20]. For the case of an integrable complex structure, i.e. when the transformation equations (5.49) and (5.50) of [15] can be integrated to become finite transformations, the metric for the mirror type IIA manifold is given by

$$
\begin{gathered}
d s_{I I A}^{2}=g_{1}\left[\left(d z-b_{z \mu} d x^{\mu}\right)+\Delta_{1} \cot \widehat{\theta}_{1}\left(d x-b_{x \theta_{1}} d \theta_{1}\right)+\Delta_{2} \cot \widehat{\theta}_{2}\left(d y-b_{y \theta_{2}} d \theta_{2}\right)+. .\right]^{2} \\
+\left[g_{2}^{\prime} d \theta_{1}^{2}+g_{2}\left(d x-b_{x \theta_{1}} d \theta_{1}\right)^{2}\right]+\left[g_{3}^{\prime} d \theta_{2}^{2}+g_{3}\left(d y-b_{y \theta_{2}} d \theta_{2}\right)^{2}\right]+\sin \psi\left[g_{4}\left(d x-b_{x \theta_{1}} d \theta_{1}\right) d \theta_{2}+\right. \\
\left.+g_{4}^{\prime}\left(d y-b_{y \theta_{2}} d \theta_{2}\right) d \theta_{1}\right]+\cos \psi\left[g_{4}^{\prime} d \theta_{1} d \theta_{2}-g_{4}\left(d x-b_{x \theta_{1}} d \theta_{1}\right)\left(d y-b_{y \theta_{2}} d \theta_{2}\right)\right]
\end{gathered}
$$

The variables appearing in the above metric are defined in 15 with a choice of $g_{4}^{\prime}, g_{2}^{\prime}$ and $g_{3}^{\prime}$ given by

$$
g_{4}^{\prime}=2 \sqrt{\langle\alpha\rangle_{1}\langle\alpha\rangle_{2}} A_{1} B_{1}, \quad g_{3}^{\prime}=\langle\alpha\rangle_{2}\left(1+A_{1}^{2}\right), \quad g_{2}^{\prime}=\langle\alpha\rangle_{1}\left(1+B_{1}^{2}\right)
$$


Observe that with this choice of integrable complex structure, we almost obtain the correct type IIA metric. In fact, we see that when we identify

$$
\langle\alpha\rangle_{1}=\langle\alpha\rangle_{2}=\alpha, \quad A_{1}=A, \quad B_{1}=B
$$

then the metric (2.4) becomes exactly the non-Kähler deformation of the deformed conifold. This can be achieved directly by choosing a non-integrable complex structure on the type IIB side (basically related to the finite transformation that we discussed in [15]).

\subsection{Background before geometric transition}

The background i.e. the metric, the $H_{N S}$ field, the coupling $g_{A}$ and the gauge field $A$, before the geometric transition can be given precisely. The metric has the following form:

$$
\begin{gathered}
d s_{I I A}^{2}=g_{1}\left[\left(d z-b_{z \mu} d x^{\mu}\right)+\Delta_{1} \cot \widehat{\theta}_{1}\left(d x-b_{x \theta_{1}} d \theta_{1}\right)+\Delta_{2} \cot \widehat{\theta}_{2}\left(d y-b_{y \theta_{2}} d \theta_{2}\right)+. .\right]^{2} \\
+g_{2}\left[d \theta_{1}^{2}+\left(d x-b_{x \theta_{1}} d \theta_{1}\right)^{2}\right]+g_{3}\left[d \theta_{2}^{2}+\left(d y-b_{y \theta_{2}} d \theta_{2}\right)^{2}\right]+g_{4} \sin \psi\left[\left(d x-b_{x \theta_{1}} d \theta_{1}\right) d \theta_{2}\right. \\
\left.+\left(d y-b_{y \theta_{2}} d \theta_{2}\right) d \theta_{1}\right]+g_{4} \cos \psi\left[d \theta_{1} d \theta_{2}-\left(d x-b_{x \theta_{1}} d \theta_{1}\right)\left(d y-b_{y \theta_{2}} d \theta_{2}\right)\right] .
\end{gathered}
$$

We see that in the absence of the $B$ fields $b_{x \theta_{1}}$ and $b_{y \theta_{2}}$ the above metric reduces to a Kähler deformed conifold, as expected. In the presence of $B$ fields the fibration structure is well defined. We will see later in other examples, that the $B$-field dependent fibration structure is universal. The three-form $H$ is given by

$$
\begin{aligned}
H_{N S} & =-\sqrt{\alpha^{3}} A(A d A+B d B) \wedge d \theta_{1} \wedge d z+\sqrt{\alpha^{3}}(A d A+B d B) \wedge d y \wedge d \theta_{2} \\
& +\sqrt{\alpha} d A \wedge d \theta_{1} \wedge d z+\sqrt{\alpha^{3}} B(A d A+B d B) \wedge\left(\sin \psi d y-\cos \psi d \theta_{2}\right) \wedge d z \\
& -\sqrt{\alpha^{3}}(A d A+B d B) \wedge d x \wedge d \theta_{1}-\sqrt{\alpha} d B \wedge\left(\sin \psi d y-\cos \psi d \theta_{2}\right) \wedge d z
\end{aligned}
$$

where $H_{N S}$ is the finite part of the three-form $\widehat{H}_{N S}$ defined as $\epsilon^{-1 / 2} H_{N S}$, with $\epsilon \rightarrow 0$. All other fields are finite. The large background three-form can be traced back to the large complex structure on the mirror type IIB side. This is of course consistent with the requirement of mirror symmetry according to which a large complex structure is related to a large base manifold (which in turn is crucial to apply the SYZ conjecture). In fact, as we will soon show, the $B_{N S}$ field when written in terms of $\langle\alpha\rangle_{1,2}$ instead of $\alpha$ becomes pure gauge and therefore the corresponding $H_{N S}$ is zero! This will have an important consequence when we go to the type IIB mirror side. 
Finally, we need to specify the gauge fields and the coupling constant. The gauge fields are associated with $D 6$ branes wrapped on a three-cycle of the non-Kähler manifold. In terms of the mirror analysis that we performed in [15] the $D 6$ branes are precisely the $D 5$ branes wrapped on the resolution $P 1$ of the Kähler resolved conifold in type IIB theory. The background now is given by

$$
A \cdot d X \equiv \Delta_{1} \cot \widehat{\theta}_{1}\left(d x-b_{x \theta_{1}} d \theta_{1}\right)-\Delta_{2} \cot \widehat{\theta}_{2}\left(d y-b_{y \theta_{2}} d \theta_{2}\right), \quad g_{A}=\frac{g_{B}}{\sqrt{1-\frac{\epsilon}{\alpha}}}
$$

and the three-form field is identically zero. This completely specifies the type IIA background before geometric transition.

\subsection{Background after geometric transition}

The background after geometric transition can also be explicitly determined. The metric now takes the following form

$$
\begin{aligned}
d s^{2}= & \frac{1}{4}\left(2 g_{2}-\frac{g_{4}}{\xi}\right)\left[d \theta_{1}^{2}+\left(d x-b_{x \theta_{1}} d \theta_{1}\right)^{2}\right]+\frac{1}{4}\left(2 g_{2}+\frac{g_{4}}{\xi}\right)\left[d \theta_{2}^{2}+\left(d y-b_{y \theta_{2}} d \theta_{2}\right)^{2}\right] \\
& +e^{2 \phi}\left[d z+\Delta_{1} \cot \widehat{\theta}_{1}\left(d x-b_{x \theta_{1}} d \theta_{1}\right)+\Delta_{2} \cot \widehat{\theta}_{2}\left(d y-b_{y \theta_{2}} d \theta_{2}\right)\right]^{2} .
\end{aligned}
$$

In the above equation we observe the familiar fibration structure again. In the absence of $b_{x \theta_{1}}$ and $b_{y \theta_{2}}$ the metric is exactly the resolved conifold metric. In the presence of the non trivial fibration the metric becomes a non-Kähler deformation of the resolved conifold. As we saw above, before the geometric transition the metric is a non-Kähler deformation of the deformed conifold. The existence of these manifolds had been anticipated earlier in the literature and their explicit form was determined in [15]. Compactifications of the type IIA theory on these backgrounds result in consistent theories.

Furthermore, the three-form $H_{N S}$ is given by

$$
\begin{aligned}
H_{N S} & =-\sqrt{\alpha^{3}} A(A d A+B d B) \wedge d \theta_{1} \wedge d z+\sqrt{\alpha^{3}}(A d A+B d B) \wedge d y \wedge d \theta_{2} \\
& +\sqrt{\alpha} d A \wedge d \theta_{1} \wedge d z+\sqrt{\alpha^{3}} B(A d A+B d B) \wedge\left(\sin \psi d y-\cos \psi d \theta_{2}\right) \wedge d z \\
& -\sqrt{\alpha^{3}}(A d A+B d B) \wedge d x \wedge d \theta_{1}-\sqrt{\alpha} d B \wedge\left(\sin \psi d y-\cos \psi d \theta_{2}\right) \wedge d z
\end{aligned}
$$

where $H_{N S}$ is the same finite part of the three-form that we had before the geometric transition took place. Thus this field is simply a spectator in this scenario and plays no other role. As discussed above, this field is exactly zero when written in terms of $\langle\alpha\rangle_{i}$, 
so we really do not have to worry about it. On the other hand, both the metric and the gauge fluxes have changed completely (along with the dilaton). The gauge field and the coupling constant are given by

$$
\mathcal{A} \cdot d X=2 \Delta_{1} \cot \widehat{\theta}_{1}\left(d x-b_{x \theta_{1}} d \theta_{1}\right), \quad g_{A}=2^{-\frac{3}{2}} e^{-\frac{\phi}{2}} \alpha^{-\frac{3}{4}}
$$

At this point the background has no $D 6$ branes anymore because these are replaced by fluxes, as expected in a geometric transition. To see explicitly that the $D 6$ branes have disappeared we need to consider how the corresponding M-theory harmonic form behaves. Some aspects of this were discussed in [15]. The existence of a normalizable M-theory harmonic form is an indication of the existence of localized gauge fluxes [46]. Similarly, for manifolds that are non-Kähler the existence of a normalizable harmonic form indicates the existence of gauge fluxes [47]. To show that such localized gauge fluxes do not exist after the geometric transition, one has to show that the harmonic forms become nonnormalizable. This analysis has still not been done explicitly, although some details have appeared in [15].

\section{Supergravity Analysis in Type IIB}

In the previous section we presented the supergravity analysis for the type IIA string before and after the geometric transition. In this section we will discuss the similar analysis for the type IIB side. The geometry on the type IIB side before the geometric transition is already known. It is a Kähler resolved conifold with $D 5$ branes wrapped on the resolution two cycle of the resolved conifold with $B_{N S}$ and $B_{R R}$ (or the corresponding field strength $H_{N S}$ and $\left.H_{R R}\right)$, turned on. The $B_{R R}$ component is related to the $D 5$ brane source. Most

of the details of this background can be extracted from [20] where this background was derived. An alternative derivation in which a fourfold with fluxes is the starting point can be found in our previous paper [15]. In the following we discuss the properties of this background.

\subsection{Background before geometric transition}

The metric and tensor fields before the geometric transition are

$$
\begin{gathered}
d s^{2}=h^{-1 / 2} d s_{0123}^{2}+h^{1 / 2}\left[\gamma^{\prime} d r^{2}+\frac{1}{4} \gamma^{\prime} r^{2}\left(d \psi+\cos \theta_{1} d \phi_{1}+\cos \theta_{2} d \phi_{2}\right)^{2}+\right. \\
\left.+\frac{1}{4} \gamma\left(d \theta_{1}^{2}+\sin ^{2} \theta_{1} d \phi_{1}^{2}\right)+\frac{1}{4}\left(\gamma+4 a^{2}\right)\left(d \theta_{2}^{2}+\sin ^{2} \theta_{2} d \phi_{2}^{2}\right)\right]
\end{gathered}
$$




$$
\begin{gathered}
B_{N S}=\mathcal{J}_{1} d \theta_{1} \wedge d x+\mathcal{J}_{2} d \theta_{2} \wedge d y, \quad F_{5}=K(r)(1+*) d x \wedge d y \wedge d z \wedge d \theta_{1} \wedge d \theta_{2} \\
H=c_{1}\left(d z \wedge d \theta_{2} \wedge d y-d z \wedge d \theta_{1} \wedge d x\right)+c_{2} \cot \widehat{\theta}_{1} d x \wedge d \theta_{2} \wedge d y-c_{3} \cot \widehat{\theta}_{2} d y \wedge d \theta_{1} \wedge d x
\end{gathered}
$$

The various quantities appearing in the above equations are defined in [20], [15]. The type IIB axion-dilaton vanishes. This background is the IR limit of the corresponding $\mathcal{N}=1$ gauge theory which is supported on the wrapped $D 5$ brane(s).

\subsection{Background after geometric transition}

In the previous section we discussed the background before geometric transition and in the following we will determine its form after geometric transition. From the duality chain presented in [15], the strategy becomes clear. One can try to construct the type IIB background as the mirror of the type IIA background after geometric transition, i.e. the mirror of (2.10). The only subtlety which arises in this approach is that on the type IIA side the isometry along the $\psi$ (or $d z$ ) direction is broken by the $B_{N S}$ fields even though all other fields preserve this background.

In order to restore the isometry in the $\psi$ direction we will perform the coordinate transformation presented in equation (4.33) of [15]. This transformation eliminates the $\psi$ dependence of $B_{N S}$. The two spheres of the type IIA metric (2.10) are invariant under this transformation. On the other hand, the $d z$ fibration is not. A similar situation appeared before in [15]. In order to solve this problem we assumed that in the delocalization limit the $\psi$ dependence in the $d z$ fibration can be easily absorbed. In this way the metric plus the complete type IIA background will have the full isometries along the $x, y$ and $z$ directions. Similarly we will assume in the present situation that after making the transformation in

equation (4.33) of 15] we recover the background with full isometries. As before, we would like to remind the readers that this is the only assumption we make.

Using this, we can write the type IIA $B_{N S}$ field as:

$$
\epsilon \frac{B_{N S}}{\sqrt{\alpha}}=d x \wedge d \theta_{1}-d y \wedge d \theta_{2}+\left(A d \theta_{1}-B d \theta_{2}\right) \wedge d z
$$

where $\alpha=\left(1+A^{2}+B^{2}\right)^{-1}$ as defined earlier. Moreover we define

$$
\alpha_{0}^{-1}=j_{x x} j_{y y}-j_{x y}^{2}=C D+\left(C B^{2}+D A^{2}\right) e^{2 \phi}
$$


with $j_{m n}$ being the components of the type IIA metric (2.10). After the mirror transformation, the type IIB metric is

$$
\begin{aligned}
d s^{2}= & \frac{1}{G_{z z}}\left(d z+\mathcal{B}_{\mu z} d x^{\mu}+\mathcal{B}_{x z} d x+\mathcal{B}_{y z} d y\right)^{2}-\frac{1}{G_{z z}}\left(G_{z \mu} d x^{\mu}+G_{z x} d x+G_{z y} d y\right)^{2} \\
& G_{\mu \nu} d x^{\mu} d x^{\nu}+2 G_{x \nu} d x d x^{\nu}+2 G_{y \nu} d y d x^{\nu}+2 G_{x y} d x d y+G_{x x} d x^{2}+G_{y y} d y^{2} .
\end{aligned}
$$

The components $G_{m n}$ are given by the following matrix elements:

$$
\begin{aligned}
G & =\left(\begin{array}{ccccc}
G_{x x} & G_{x y} & G_{x z} & G_{x \theta_{1}} & G_{x \theta_{2}} \\
G_{x y} & G_{y y} & G_{y z} & G_{y \theta_{1}} & G_{y \theta_{2}} \\
G_{x z} & G_{y z} & G_{z z} & G_{z \theta_{1}} & G_{z \theta_{2}} \\
G_{x \theta_{1}} & G_{y \theta_{1}} & G_{z \theta_{1}} & G_{\theta_{1} \theta_{1}} & G_{\theta_{1} \theta_{2}} \\
G_{x \theta_{2}} & G_{y \theta_{2}} & G_{z \theta_{2}} & G_{\theta_{1} \theta_{2}} & G_{\theta_{2} \theta_{2}}
\end{array}\right) \\
= & \left(\begin{array}{ccccc}
\alpha_{0} D_{1} & -\alpha_{0} e^{2 \phi} A B & 0 & -\alpha_{0} \sqrt{\alpha} D_{1} & -\alpha_{0} \sqrt{\alpha} e^{2 \phi} A B \\
-\alpha_{0} e^{2 \phi} A B & \alpha_{0} C_{1} & 0 & \alpha_{0} \sqrt{\alpha} e^{2 \phi} A B & \alpha_{0} \sqrt{\alpha} C_{1} \\
0 & 0 & \alpha_{0} e^{2 \phi} C D & 0 & 0 \\
-\alpha_{0} \sqrt{\alpha} D_{1} & \alpha_{0} \sqrt{\alpha} e^{2 \phi} A B & 0 & C+\alpha_{0} \sqrt{\alpha} D_{1} & \alpha_{0} \alpha e^{2 \phi} A B \\
-\alpha_{0} \sqrt{\alpha} e^{2 \phi} A B & \alpha_{0} \sqrt{\alpha} C_{1} & 0 & \alpha_{0} \alpha e^{2 \phi} A B & D+\alpha_{0} \alpha C_{1}
\end{array}\right)
\end{aligned}
$$

where $A$ and $B$ have been defined in [15] and $C$ and $D$ were related to the mirror type IIA metric:

$$
\begin{aligned}
& C=\frac{g_{2}}{2}-\frac{g_{4}}{4 \xi}=\frac{\alpha}{2}\left(1+B^{2}-\frac{A B}{\sqrt{\left(1+A^{2}\right) /\left(1+B^{2}\right)}}\right) \\
& D=\frac{g_{2}}{2}+\frac{g_{4}}{4 \xi}=\frac{\alpha}{2}\left(1+B^{2}+\frac{A B}{\sqrt{\left(1+A^{2}\right) /\left(1+B^{2}\right)}}\right) .
\end{aligned}
$$

$C_{1}$ and $D_{1}$ are short hand notation for

$$
C_{1}=C+A^{2} e^{2 \phi}, \quad D_{1}=D+B^{2} e^{2 \phi} .
$$

Using the above form of the metric components (3.7), the $d z$ fibration structure can be shown to take the following form:

$$
\begin{gathered}
\frac{1}{G_{z z}}\left[d z-A\left[\alpha_{0} e^{2 \phi} D d x-\epsilon^{-1} \sqrt{\alpha}\left(1+\alpha_{0} e^{2 \phi} D\right) d \theta_{1}\right]\right. \\
\left.-B\left[\alpha_{0} e^{2 \phi} C d y+\epsilon^{-1} \sqrt{\alpha}\left(1+\alpha_{0} e^{2 \phi} C\right) d \theta_{2}\right]\right]^{2} .
\end{gathered}
$$


The $d z$ fibration structure can be re-grouped so that it takes a simpler form, in the following way

$$
\begin{aligned}
& \frac{1}{G_{z z}}\left[\left(d z+A \epsilon^{-1} \sqrt{\alpha} d \theta_{1}-B \epsilon^{-1} \sqrt{\alpha} d \theta_{2}\right)-\right. \\
& \left.\quad A \alpha_{0} e^{2 \phi} D\left(d x-\epsilon^{-1} \sqrt{\alpha} d \theta_{1}\right)-B \alpha_{0} e^{2 \phi} C\left(d y+\epsilon^{-1} \sqrt{\alpha} d \theta_{2}\right)\right]^{2} .
\end{aligned}
$$

The fibration structure has many interesting properties. First, the $d x$ and the $d y$ directions are fibered over the base in the expected way due to the background $B_{N S}$ fields on the type IIA side. The $d x, d y$ fibrations are

$$
d x-\epsilon^{-1} \sqrt{\alpha} d \theta_{1}, \quad d y+\epsilon^{-1} \sqrt{\alpha} d \theta_{2}
$$

Does this mean that in type IIB theory we get again a non-Kähler manifold in the closed string side? Furthermore, due to small $\epsilon$, the non-Kählerity would be very large!

Fortunately, this is not the case as the fibration structure that we see above actually cancels the transformations that we did earlier in the open string side of type IIB to get the mirror type IIA manifolds! Thus even though there are non-trivial fibrations, these are actually quite harmless and therefore will not produce any non-Kählerity in the manifold. Thus we seem to recover a Kähler manifold (at least at the fibration level) in the type IIB picture. This is again supported by the other components of the metric which are:

$$
\begin{aligned}
d s^{2}= & {\left[C d \theta_{1}^{2}+\alpha_{0} D_{1}\left(d x-\epsilon^{-1} \sqrt{\alpha} d \theta_{1}\right)^{2}\right]+\left[D d \theta_{2}^{2}+\alpha_{0} C_{1}\left(d y+\epsilon^{-1} \sqrt{\alpha} d \theta_{2}\right)^{2}\right] } \\
& +\left[0 \cdot d \theta_{1} d \theta_{2}-2 \alpha_{0} A B e^{2 \phi}\left(d x-\epsilon^{-1} \sqrt{\alpha} d \theta_{1}\right)\left(d y+\epsilon^{-1} \sqrt{\alpha} d \theta_{2}\right)\right] .
\end{aligned}
$$

Looking carefully at the metric we see that our old problem has come back to haunt us again. The metric has no $d \theta_{1} d \theta_{2}$ factor. Whatever $d \theta_{1} d \theta_{2}$ factors were created via mirror transformations have gone into the fibration. This means that the type IIA manifold that we started with doesn't really suffice, and we need to put a non-trivial complex structure on it before we even start doing the mirror operation.

In the following we will discuss the three parts of the metric, $z$-fibration and the two lines in (3.13), separately. Let us denote the three terms by

$$
d s_{I I B}^{2}=d s_{z}^{2}+d s_{T}^{2}+d s_{12}^{2},
$$

where $d s_{z}^{2}$ stands for the $z$-fibration, $d s_{T}^{2}$ refers to the first line in (3.13), coming from the two tori defined below, and $d s_{12}^{2}$ describes the $\theta_{1}-\theta_{2}$ cross terms. 
Let us define two tori with complex structures $\tau_{1}$ and $\tau_{2}$ in the following way:

$$
d z_{1} \equiv d x-\tau_{1} d \theta_{1}, \quad d z_{2} \equiv d y-\tau_{2} d \theta_{2}
$$

where $\tau_{1}$ and $\tau_{2}$ are of the form:

$$
\tau_{1}=f_{1}+b_{x \theta_{1}}+i, \quad \tau_{2}=f_{2}+b_{y \theta_{2}}+i
$$

Here, $f_{1}$ and $f_{2}$ are still arbitrary and will be fixed below. The type IIA metric (2.10) can now be written with non-trivial complex structures, as $d s^{2}=j_{m n} d x^{m} d x^{n}$, with $j_{m n}$ being given by:

$$
\begin{aligned}
j=\left(\begin{array}{ccccc}
j_{x x} & j_{x y} & j_{x z} & j_{x \theta_{1}} & j_{x \theta_{2}} \\
j_{x y} & j_{y y} & j_{y z} & j_{y \theta_{1}} & j_{y \theta_{2}} \\
j_{x z} & j_{y z} & j_{z z} & j_{z \theta_{1}} & j_{z \theta_{2}} \\
j_{x \theta_{1}} & j_{y \theta_{1}} & j_{z \theta_{1}} & j_{\theta_{1} \theta_{1}} & j_{\theta_{1} \theta_{2}} \\
j_{x \theta_{2}} & j_{y \theta_{2}} & j_{z \theta_{2}} & j_{\theta_{1} \theta_{2}} & j_{\theta_{2} \theta_{2}}
\end{array}\right) \\
=\left(\begin{array}{ccccc}
C_{1} & e^{2 \phi} A B \\
e^{2 \phi} A B & D_{1} & e^{2 \phi} A & -b_{x \theta_{1}} C_{1}-f_{1} C & -e^{2 \phi} b_{y \theta_{2}} A B \\
e^{2 \phi} A & & e^{2 \phi} B & -e^{2 \phi} b_{x \theta_{1}} A B & -b_{y \theta_{2}} D_{1}-f_{2} D \\
-b_{x \theta_{1}} C_{1}-f_{1} C & -e^{2 \phi} b_{y \theta_{2}} A B & -e^{2 \phi} b_{x \theta_{1}} A & C F_{1}+b_{x \theta_{1}}^{2} C_{1} & e^{2 \phi} A B b_{x \theta_{1}} b_{y \theta_{2}} \\
-e^{2 \phi} b_{y \theta_{2}} A B & -b_{y \theta_{2}} D_{1}-f_{2} D & -e^{2 \phi} b_{y \theta_{2}} B & e^{2 \phi} A B b_{x \theta_{1}} b_{y \theta_{2}} & D F_{2}+b_{y \theta_{2}}^{2} D_{1}
\end{array}\right)
\end{aligned}
$$

$F_{1}$ and $F_{2}$ used in the above metric are defined as follows:

$$
F_{1}=1+f_{1}^{2}+2 f_{1} b_{x \theta_{1}}, \quad F_{2}=1+f_{2}^{2}+2 f_{2} b_{y \theta_{2}}
$$

and the other quantities have been defined in [15].

In the above matrix components, observe that we have again shifted the $j_{z z}$ components by $\epsilon$, i.e.

$$
j_{z z}=\lim _{\epsilon \rightarrow 0}\left(e^{2 \phi}-\epsilon\right) .
$$

The two spheres (which we have now taken as two tori) have the following metric

$$
d s_{T(I I A)}^{2}=C\left|d z_{1}\right|^{2}+D\left|d z_{2}\right|^{2}
$$


with $C, D$ defined above. The $d z$ fibration structure changes as (3.19).

After the mirror transformation on the metric (3.17), some of the components of the mirror metric are not affected by the non-trivial complex structure. They are

$$
G_{x x}=\alpha_{0} j_{y y}=\alpha_{0}\left(D+e^{2 \phi} B^{2}\right), \quad G_{y y}=\alpha_{0} j_{x x}=\alpha_{0}\left(C+e^{2 \phi} A^{2}\right)
$$

and also the other two components:

$$
G_{x \theta_{1}}=\alpha_{0}\left(B_{y \theta_{1}} j_{x y}-B_{x \theta_{1}} j_{y y}\right) \quad G_{x \theta_{2}}=\alpha_{0}\left(B_{y \theta_{2}} j_{x y}-B_{x \theta_{2}} j_{y y}\right)
$$

and similarly $G_{y \theta_{1}}$ and $G_{y \theta_{2}}$. Here $B_{m n}$ denote the $B_{N S}$ fields as it appeared earlier in (3.4). With the choice of an integrable complex structure in type IIB before geometric transition, the $B_{N S}$ fields take the following form:

$$
\epsilon B_{N S}=\sqrt{\langle\alpha\rangle_{1}} d x \wedge d \theta_{1}-\sqrt{\langle\alpha\rangle_{2}} d y \wedge d \theta_{2}+\left(A \sqrt{\langle\alpha\rangle_{1}} d \theta_{1}-B \sqrt{\langle\alpha\rangle_{2}} d \theta_{2}\right) \wedge d z
$$

where $\langle\alpha\rangle_{i}$ have already been defined in sec. 2 . One can now easily see that this $B$ field is actually a pure gauge! In other words $B_{N S}=d \Lambda$. We will derive the form of $\Lambda$ below.

Our next goal is to get a non-vanishing $d \theta_{1} d \theta_{2}$ term in the metric component $d s_{12}^{2}$. According to the arguments of [15], this can be achieved by using a non-trivial complex structure. However, the metric on the type IIA side (2.10) is much more involved since it is non-Kähler. Therefore, it is necessary to go through the whole argument once again to check if it applies to the present case.

Using a complex structure more complicated than $\tau=i$ will result in non-vanishing metric components $G_{z \theta_{i}}$ etc. As a consequence the metric along the $\theta_{i} \theta_{j}(i, j=1,2)$ directions will have the following form (excluding the $B$-dependent terms which go into the $z$-fibration):

$$
d s_{\theta_{i} \theta_{j}}^{2}=\left(G_{\theta_{i} \theta_{j}}-\frac{G_{z \theta_{i}} G_{z \theta_{j}}}{G_{z z}}\right) d \theta_{i} d \theta_{j} .
$$

Let us compute this term by term. First we consider the $G_{z z}$ component. Choosing $j_{z z}=e^{2 \phi}-\epsilon$, we obtain:

$$
G_{z z}=j_{z z}-\alpha_{0}\left[j_{y y} j_{x z}^{2}+j_{x x} j_{y z}^{2}-2 j_{x z} j_{y z} j_{x y}\right]=\alpha_{0} C D e^{2 \phi}-\epsilon .
$$

The $\epsilon$ dependence is expected from the analysis of [15]. However, the finite and non-zero part is more involved than the one of [15]. As a result all the subsequent components 
will become more involved because of the non-Kähler nature of the starting manifold. Similarly the $G_{z \theta_{i}}$ component is

$$
G_{z \theta_{i}}=j_{z \theta_{i}}-\alpha_{0}\left[j_{x \theta_{i}} j_{x z} j_{y y}+j_{y \theta_{i}} j_{y z} j_{x x}-j_{x y}\left(j_{y \theta_{i}} j_{x z}+j_{x \theta_{i}} j_{y z}\right)\right]
$$

For $i=1,2$ one obtains

$$
G_{z \theta_{1}}=\alpha_{0} f_{1} e^{2 \phi} C A D, \quad G_{z \theta_{2}}=\alpha_{0} f_{2} e^{2 \phi} D B C
$$

Observe that $f_{1}=f_{2}=0$ implies a vanishing of $G_{z \theta_{1}}$ and $G_{z \theta_{2}}$. This is consistent with what we already know from our earlier analysis.

The other components, like $G_{x x}, G_{y y}$ for example, remain unchanged from their values with trivial complex structure of $\tau=i$. In the following we will compute $G_{\theta_{1} \theta_{2}}$. Using mirror symmetry this component is given by

$G_{\theta_{1} \theta_{2}}=j_{\theta_{1} \theta_{2}}-\alpha_{0} j_{x y} B_{x \theta_{1}} B_{y \theta_{2}}-\alpha_{0}\left[j_{y y} j_{x \theta_{1}} j_{x \theta_{2}}+j_{x x} j_{y \theta_{1}} j_{y \theta_{2}}-j_{x y}\left(j_{y \theta_{1}} j_{x \theta_{2}}+j_{x \theta_{1}} j_{y \theta_{2}}\right)\right]$.

The above relation is a special case of results obtained previously in [15]. In deriving (3.28) we have inherently assumed a particular form for the $B_{N S}$ field, namely $B_{x \theta_{2}}=B_{y \theta_{1}}=0$. However, the cross components are non-vanishing. This is where (3.28) would differ from the equivalent formula that we had in the type IIB case of [15]. The existence of non-trivial cross terms is related to non-Kählerity as well as non-trivial complex structures of the two tori. For $\tau=i$, the above relation turns into

$$
G_{\theta_{1} \theta_{2}}^{\text {old }}=-\alpha_{0} j_{x y} B_{x \theta_{1}} B_{y \theta_{2}}
$$

where we have assumed $f_{i}=0$. Including non-vanishing values for $f_{1}$ and $f_{2}$ results in a simple extension of our previous results in [15], i.e.:

$$
G_{\theta_{1} \theta_{2}}=-\alpha_{0} j_{x y} B_{x \theta_{1}} B_{y \theta_{2}}+\alpha_{0} e^{2 \phi} A B C D f_{1} f_{2}
$$

To obtain the final answer we still have to compute the $G_{z \theta_{i}}$ terms as in (3.24) (for $i=1$ and $j=2$ ), and we need to determine the functions $f_{1}$ and $f_{2}$. The relation (3.24) implies

$$
d s_{\theta_{1} \theta_{2}}^{2}=-2\left(\alpha_{0} j_{x y} B_{x \theta_{1}} B_{y \theta_{2}}+f_{1} f_{2} A B \epsilon\right) d \theta_{1} d \theta_{2}
$$


Now we are close to obtaining the final expression for $d s_{12}^{2}$. The first term in (3.31) is the usual term we discussed before. The second term is important. We want the single $\theta_{1}-\theta_{2}$ cross term, that does not arise from any fibration, to have the same pre-factor as the $x-y$ cross term from the metric obtained with vanishing $f_{1}$ and $f_{2}$. This is achieved by choosing $f_{1}, f_{2}$ to satisfy the following relation:

$$
f_{1} f_{2}=-\epsilon^{-1} \alpha_{0} e^{2 \phi}
$$

This is our first non trivial conclusion. The above relation is reminiscent to a result obtained previously in [15]. If we set $f_{i} \equiv \pm \epsilon^{-1 / 2} \beta_{i}$, then $\beta_{1} \beta_{2}=\alpha_{0} e^{2 \phi}$. With this choice, the metric for the $\theta_{1} \theta_{2}$ direction becomes simple, and is given by

$$
d s_{\theta_{1} \theta_{2}}^{2}=-2 \alpha_{0}\left(j_{x y} B_{x \theta_{1}} B_{y \theta_{2}}-e^{2 \phi} A B\right) d \theta_{1} d \theta_{2}=-2 \alpha_{0}\left(j_{x y} B_{x \theta_{1}} B_{y \theta_{2}}-j_{x y}\right) d \theta_{1} d \theta_{2} .
$$

The last identification stems from the type IIA metric, and should remind the reader of a similar coefficient in the type IIA case before geometric transition. Finally, the first term in (3.33) can be combined with the metric components in the $x, y$ direction to give us the complete metric for the cross terms:

$$
d s_{12}^{2}=2 \alpha_{0} j_{x y}\left[d \theta_{1} d \theta_{2}-\left(d x-\epsilon^{-1} \sqrt{\langle\alpha\rangle_{1}} d \theta_{1}\right)\left(d y+\epsilon^{-1} \sqrt{\langle\alpha\rangle_{2}} d \theta_{2}\right)\right]
$$

Here we have substituted the values of the $B$ fields in (3.23) to get the fibration structure in the desired form. This is the full structure of the cross terms in the metric.

In the following we will obtain the fibration structure in the $d x$ and $d y$ directions. As discussed above, this non-trivial fibration is rather harmless because when (3.34) is written in terms of original type IIB coordinates before the geometric transition, the metric is Kähler. To make this discussion precise, let us rewrite the $d x$ fibration more suggestively (a similar argument will go through for the $d y$ coordinate). First, we need the functional form for $\langle\alpha\rangle_{1}$. This can be obtained from appendix 1 of 15

$$
\langle\alpha\rangle_{1}=\frac{1}{1+\Delta_{1}^{2} \cot ^{2} \theta_{1}+\Delta_{2}^{2} \cot ^{2}\left\langle\theta_{2}\right\rangle} \equiv \frac{1}{a+b \cot ^{2} \theta_{1}} .
$$

Here $\left\langle\theta_{2}\right\rangle, a$ and $b$ are taken to be constants, and we have already defined $\Delta_{i}$. These are all constants for the case considered in [15], $\delta r=0$. 
Using the above form of $\langle\alpha\rangle_{1}$, we will rewrite the $d x$ fibration structure. We will assume that $b>a$. The $b<a$ case can be treated similarly. The fibration structure takes the following form:

$$
\begin{aligned}
d \widehat{x} & \equiv d x-\epsilon^{-1} \sqrt{\langle\alpha\rangle_{1}} d \theta_{1} \\
& =d\left[x+\frac{\epsilon^{-1} \sqrt{a}}{\sqrt{b-a}} \ln \left(\sqrt{\frac{b-a}{a}} \cos \theta_{1}+\sqrt{\cos ^{2} \theta_{1}-\frac{b}{a} \sin ^{2} \theta_{1}}\right)\right]
\end{aligned}
$$

where $\widehat{x}$ is related to the coordinate that we started with on the resolved conifold side before the geometric transition. After a simple sign change the $d y$ fibration can be treated similarly. The final result for the metric is

$$
d s_{12}^{2}=2 \alpha_{0} A B e^{2 \phi}\left(d \theta_{1} d \theta_{2}-d \widehat{x} d \widehat{y}\right) .
$$

For completeness, let us also define

$$
d \widehat{z}=d z+\epsilon^{-1} A \sqrt{\alpha} d \theta_{1}-\epsilon^{-1} B \sqrt{\alpha} d \theta_{2} .
$$

In order to obtain the final result for the metric we still have to determine $f_{1}$ and $f_{2}$ (or equivalently, $\beta_{1}, \beta_{2}$ ). This can be done using the reasoning laid out in [15]. The functions $f_{1}, f_{2}$ can be determined from the metric of the two spheres (or equivalently, two tori, as we did everything assuming them to be tori). Let us first consider the torus described by the coordinates $\left(\theta_{2}, y\right)$. For a trivial complex structure on the type IIA side the metric along the tori direction can be extracted from (3.13). For non-vanishing $f_{2}$, the $G_{\theta_{2} \theta_{2}}$ component is

$$
G_{\theta_{2} \theta_{2}}=j_{\theta_{2} \theta_{2}}+\alpha_{0}\left[j_{x x} B_{y \theta_{2}}^{2}-j_{y y} j_{x \theta_{2}}^{2}-j_{x x} j_{x \theta_{2}}^{2}+2 j_{x y} j_{x \theta_{2}} j_{y \theta_{2}}\right]
$$

After using a non-vanishing value for $f_{2}$, terms proportional to $f_{2}$ and $f_{2}^{2}$ appear. As a result one obtains

$$
G_{\theta_{2} \theta_{2}}=\mathcal{A}_{0} f_{2}^{0}+\mathcal{A}_{1} f_{2}+\mathcal{A}_{2} f_{2}^{2}
$$

which terminates at this order because of the mirror formula (3.39). As it is also clear that there would be no negative powers of $f_{2}$. A similar analysis can be performed for the component $G_{\theta_{1} \theta_{1}}$, where the series will be determined by $f_{1}$. The coefficients $\mathcal{A}_{i}$ are

$$
\begin{aligned}
& \mathcal{A}_{0}=D+\alpha_{0}\left(C+e^{2 \phi} A^{2}\right) B_{y \theta_{2}}^{2}, \quad \mathcal{A}_{2}=D-\alpha_{0}\left(C+e^{2 \phi} B^{2}\right) D^{2} \\
& \mathcal{A}_{1}=2 D b_{y \theta_{2}}-2 D \alpha_{0}\left(D+e^{2 \phi} B^{2}\right)\left(C+e^{2 \phi} A^{2}\right) b_{y \theta_{2}}+2 D \alpha_{0} A^{2} B^{2} e^{4 \phi} b_{y \theta_{2}}
\end{aligned}
$$


Let us consider these coefficients carefully. The first coefficient $\mathcal{A}_{0}$ also appeared for a trivial complex structure. The second coefficient $\mathcal{A}_{1}$ can be shown to vanish. The third coefficient $\mathcal{A}_{2}$ simplifies after substituting the values for $\alpha_{0}$ etc. As a result the additional terms in $G_{\theta_{2} \theta_{2}}$ for non zero $f_{2}$ can now be concisely written as:

$$
\mathcal{A}_{2}=\alpha_{0} C D B^{2} e^{2 \phi}, \quad \mathcal{A}_{1}=0
$$

The above is of course not the complete answer, as the metric along the $\theta_{2} \theta_{2}$ direction is also influenced by $G_{z \theta_{2}}$. We will evaluate this contribution below. But first, let us consider the other component $G_{\theta_{1} \theta_{1}}$. This is written in terms of $f_{1}$ with coefficients $\mathcal{B}_{i}, i=0,1,2$, given by

$$
\mathcal{B}_{0}=C+\alpha_{0}\left(D+e^{2 \phi} B^{2}\right) B_{x \theta_{1}}^{2}, \quad \mathcal{B}_{1}=0, \quad \mathcal{B}_{2}=\alpha_{0} C D A^{2} e^{2 \phi} .
$$

Now we are getting closer to the final result. The metric along the $\theta_{1}^{2}$ or the $\theta_{2}^{2}$ directions will get contributions from $G_{z \theta_{i}}$ and $G_{z z}$. This implies (again excluding the contributions that are absorbed into the $z$-fibration $d s_{z}^{2}$ ):

$$
\begin{aligned}
& d s_{\theta_{1} \theta_{1}}^{2}=C+\epsilon^{-2} \alpha_{0} \alpha\left(D+e^{2 \phi} B^{2}\right)-\beta_{1}^{2} A^{2} \\
& d s_{\theta_{2} \theta_{2}}^{2}=D+\epsilon^{-2} \alpha_{0} \alpha\left(C+e^{2 \phi} A^{2}\right)-\beta_{2}^{2} B^{2} .
\end{aligned}
$$

In order to complete our result we still need to determine $\beta_{1}$ and $\beta_{2}$. We take the results of [15] as a guide. Requiring the terms $d s_{\theta_{2} \theta_{2}}^{2}$ and the $y y$ component of the metric to be equal, we can perform the coordinate transformation (4.33) of [15]. After this transformation the metric takes the deformed conifold form. Therefore to get the value of $\beta_{2}$ we have to identify some parts of $d s_{\theta_{2} \theta_{2}}^{2}$ with $G_{y y}$. The part of $d s_{\theta_{2} \theta_{2}}^{2}$ that is relevant is of course the one that does not go into the $d y$ fibration structure, i.e. that independent of $b_{y \theta_{2}}$ or $\alpha$ in (3.44). In other words, we have

$$
D-\beta_{2}^{2} B^{2}=\alpha_{0}\left(C+e^{2 \phi} A^{2}\right) .
$$

Combining the above equation with (3.32) determines both $\beta_{1}$ and $\beta_{2}$. They result is

$$
\begin{aligned}
& \beta_{1}=\frac{\sqrt{\alpha_{0}} e^{2 \phi}}{\sqrt{e^{2 \phi} C D-\frac{\left(C+e^{2 \phi} A^{2}\right)\left(1-D^{2}\right)}{B^{2}}}} \\
& \beta_{2}=\sqrt{\alpha_{0} e^{2 \phi} C D-\frac{\alpha_{0}\left(C+e^{2 \phi} A^{2}\right)\left(1-D^{2}\right)}{B^{2}}}
\end{aligned}
$$


The above functional form for $\beta_{2}$ shows that the coefficients of $d s_{\theta_{2} \theta_{2}}^{2}$ and $G_{y y}$ are the same. On the other hand, the functional form for $\beta_{1}$ implies that the coefficients of $d s_{\theta_{1} \theta_{1}}^{2}$ and $G_{x x}$ are not same. In the case studied in [15] the coefficients for both the tori were made equal by going to a canonical basis. The choice of the canonical basis was possible there because we took the case where $\delta r=0$ and therefore the coefficients for the tori metric were basically constants and could be absorbed in the definition of $d \theta_{i}$. Here, however, the metric of the tori in type IIA have coefficients that are functions of all the coordinates. So a simple redefinition cannot be made; implying that we can bring only one of the tori into the desired form. This causes no problem of course, because we are anyway making coordinate transformation (i.e the transformation equation 4.33 of [15]) to one of the tori.

The final result for the type IIB metric is

$$
\begin{array}{r}
d s^{2}=h_{1}\left[d \widehat{z}+a_{1} d \widehat{x}+a_{2} d \widehat{y}\right]^{2}+h_{2}\left[d \widehat{y}^{2}+d \theta_{2}^{2}\right]+h_{4}\left[d \widehat{x}^{2}+h_{3} d \theta_{1}^{2}\right]+ \\
+h_{5} \sin \psi\left[d \widehat{x} d \theta_{2}+d \widehat{y} d \theta_{1}\right]+h_{5} \cos \psi\left[d \theta_{1} d \theta_{2}-d \widehat{x} d \widehat{y}\right]
\end{array}
$$

with the $h_{i}$ defined below. This should be a Kähler manifold as it lacks the $B$ field dependent fibration structure, which made the type IIA manifold non-Kähler. This metric is similar to the Klebanov-Strassler [1] background. In fact, there is a simple reason for this. The $B_{N S}$ field we discussed on the type IIA side in (3.23) is pure gauge and can be written in terms of $d \Lambda$. Defining $\Delta=\sqrt{1-k^{2} \sin ^{2} \theta_{1}}$ and $k=\sqrt{\frac{b-a}{b}}$, we see that

$$
\begin{aligned}
& \sqrt{\langle\alpha\rangle_{1}} d x \wedge d \theta_{1}=\frac{1}{k \sqrt{b}} d\left[\ln \left(k \cos \theta_{1}+\Delta\right)\right] \wedge d x \\
& A \sqrt{\langle\alpha\rangle_{1}} d \theta_{1} \wedge d z=\frac{1}{k \sqrt{b}} d\left[\sqrt{b} \arctan \frac{k \sin \theta_{1}}{\Delta}\right] \wedge d z
\end{aligned}
$$

with similar relations for the $\theta_{2}$ coordinates. From the above relation we see that $\Lambda$ can be defined as follows:

$$
\Lambda=\left[\ln \left(k \cos \theta_{1}+\Delta\right)\right] \wedge d x+\left[\sqrt{b} \arctan \frac{k \sin \theta_{1}}{\Delta}\right] \wedge d z-\left\{\theta_{1} \leftrightarrow \theta_{2}, x \leftrightarrow y\right\}
$$

up to an overall constant factor. Since the $B_{N S}$ field can be gauged away, the type IIB metric remains Kähler. On the other hand, the original type IIB $B_{N S}$ field given in [20], [15] is not a gauge artifact and therefore does make the type IIA manifolds non-Kähler both before and after geometric transition. 
The two tori appearing in the type IIB metric have complex structures $\tau_{2}=i$ and $\tau_{1}=i \sqrt{h_{3}}$. Using this we can define the corresponding metric as

$$
d s^{2}=h_{2}\left|d z_{2}\right|^{2}+h_{4}\left|d z_{1}\right|^{2}, \quad d z_{2}=d \widehat{y}+i d \theta_{2}, \quad d z_{1}=d \widehat{x}+i \sqrt{h_{3}} d \theta_{1} .
$$

Here we have used the notation

$$
\begin{gathered}
h_{1}=\frac{e^{-2 \phi}}{\alpha_{0} C D}, \quad h_{2}=\alpha_{0}\left(C+e^{2 \phi} A^{2}\right), \quad h_{4}=\alpha_{0}\left(D+e^{2 \phi} B^{2}\right), \quad h_{5}=2 \alpha_{0} e^{2 \phi} A B \\
h_{3}=\frac{C-\beta_{1}^{2} A^{2}}{\alpha_{0}\left(D+e^{2 \phi} B^{2}\right)}, \quad a_{1}=-\alpha_{0} e^{2 \phi} A D, \quad a_{2}=-\alpha_{0} e^{2 \phi} B C
\end{gathered} .
$$

So far we have been mostly concentrated on deriving the background metric. In the following we will determine other background fields, like $B_{N S}, B_{R R}$, the axion and the dilaton. We will first consider the $B_{N S}$ field. After applying mirror symmetry, $B_{N S}$ is found to be:

$$
\begin{aligned}
B^{b}= & \left(\tilde{B}_{\mu \nu}+\frac{2 \tilde{B}_{z[\mu} G_{\nu] z}}{G_{z z}}\right) d x^{\mu} \wedge d x^{\nu}+\left(\tilde{B}_{\mu x}+\frac{2 \tilde{B}_{z[\mu} G_{x] z}}{G_{z z}}\right) d x^{\mu} \wedge d x \\
& \left(\tilde{B}_{\mu y}+\frac{2 \tilde{B}_{z[\mu} G_{y] z}}{G_{z z}}\right) d x^{\mu} \wedge d y+\left(\tilde{B}_{x y}+\frac{2 \tilde{B}_{z[x} G_{y] z}}{G_{z z}}\right) d x \wedge d y \\
& +\frac{G_{z \mu}}{G_{z z}} d x^{\mu} \wedge d z+\frac{G_{z x}}{G_{z z}} d x \wedge d z+\frac{G_{z y}}{G_{z z}} d y \wedge d z .
\end{aligned}
$$

The above relation has already been derived in [15]. In the present case we have to take into account that before T-duality the $B_{N S}$ field (3.23) changes due to the introduction of a non-trivial complex structure in (3.16):

$$
\epsilon \frac{B_{N S}}{\sqrt{\alpha}}=d x \wedge d \theta_{1}-d y \wedge d \theta_{2}+\left(A d \theta_{1}-B d \theta_{2}\right) \wedge d z+A B\left(f_{1}+f_{2}\right) d \theta_{1} \wedge d \theta_{2} .
$$

Here we have taken the limit depicted earlier in (2.6) i.e. $\langle\alpha\rangle_{1}=\langle\alpha\rangle_{2}=\alpha$. If we do not consider this limit then this will again be a gauge artifact. The various components of $\tilde{B}$ in the above relation (3.52) become

$$
\tilde{B}_{N S}=\left(\begin{array}{ccccc}
\tilde{B}_{x x} & \tilde{B}_{x y} & \tilde{B}_{x z} & \tilde{B}_{x \theta_{1}} & \tilde{B}_{x \theta_{2}} \\
\tilde{B}_{y x} & \tilde{B}_{y y} & \tilde{B}_{y z} & \tilde{B}_{y \theta_{1}} & \tilde{B}_{y \theta_{2}} \\
\tilde{B}_{z x} & \tilde{B}_{z y} & \tilde{B}_{z z} & \tilde{B}_{z \theta_{1}} & \tilde{B}_{z \theta_{2}} \\
\tilde{B}_{\theta_{1} x} & \tilde{B}_{\theta_{1} y} & \tilde{B}_{\theta_{1} z} & \tilde{B}_{\theta_{1} \theta_{1}} & \tilde{B}_{\theta_{1} \theta_{2}} \\
\tilde{B}_{\theta_{2} x} & \tilde{B}_{\theta_{2} y} & \tilde{B}_{\theta_{2} z} & \tilde{B}_{\theta_{2} \theta_{1}} & \tilde{B}_{\theta_{2} \theta_{2}}
\end{array}\right)
$$




$$
=\left(\begin{array}{ccccc}
0 & 0 & -\alpha_{0} e^{2 \phi} A D & b_{x} \theta_{1} E_{3} & -\alpha_{0} e^{2 \phi} A B D f_{2} \\
0 & 0 & -\alpha_{0} e^{2 \phi} B C & -\alpha_{0} e^{2 \phi} A B C f_{1} & b_{y \theta_{2}} E_{4} \\
\alpha_{0} e^{2 \phi} A D & \alpha_{0} e^{2 \phi} B C & 0 & -\epsilon^{-1} A \sqrt{\alpha} E_{2} & \epsilon^{-1} B \sqrt{\alpha} E_{1} \\
-b_{x \theta_{1}} E_{3} & \alpha_{0} e^{2 \phi} A B C f_{1} & \epsilon^{-1} A \sqrt{\alpha} E_{2} & 0 & \epsilon^{-1} \sqrt{\alpha} A B F_{3} \\
\alpha_{0} e^{2 \phi} A B D f_{2} & -b_{y \theta_{2}} E_{4} & -\epsilon^{-1} B \sqrt{\alpha} E_{1} & -\epsilon^{-1} \sqrt{\alpha} A B F_{3} & 0
\end{array}\right) .
$$

Here we have defined the quantities $E_{i}$ and $F_{3}$ in terms of $C_{1}$ and $D_{1}$ as follows:

$$
\begin{array}{ll}
E_{1}=1+\alpha_{0} e^{2 \phi} C, & E_{3}=1+\frac{\alpha_{0} f_{1} C D_{1}}{b_{x \theta_{1}}} \\
E_{2}=1+\alpha_{0} e^{2 \phi} D, & E_{4}=1+\frac{\alpha_{0} f_{2} D C_{1}}{b_{y \theta_{2}}} \\
F_{3}=f_{1} E_{1}+f_{2} E_{2} . &
\end{array}
$$

Substituting (3.54) in (3.52) we find that the $\theta_{1} \theta_{2}$ component of the $B$ field, $B_{\theta_{1} \theta_{2}}^{b}$, vanishes. Note, that this could not have been achieved without taking the change in the $B$-field (3.53) into account. The type IIB $B_{N S}$ becomes:

$$
B^{b}=\left(b_{x \theta_{1}}+f_{1}\right) d x \wedge d \theta_{1}+\left(b_{y \theta_{2}}+f_{2}\right) d y \wedge d \theta_{2}+\left(A f_{1} d \theta_{1}+B f_{2} d \theta_{2}\right) \wedge d z
$$

Observe that the above structure of the B-field might look different from the original type IIB field we considered in [15] before the geometric transition. This is due to the nonzero values for the $z$ components of the original type IIA B-field and also the underlying non-Kähler nature of the type IIA background. The non-Kähler nature which arises due to the non-trivial fibrations in the $x, y$ and $z$ directions causes cross terms in the type IIA metric. These cross terms eventually become the $B$ fields on the type IIB side. However, as we discussed before, if the $B$ field components with coefficients $f_{1}, f_{2}$ are pure gauge, the final result will be simply given by

$$
B^{b}=b_{x \theta_{1}} d \widehat{x} \wedge d \theta_{1}+b_{y \theta_{2}} d \widehat{y} \wedge d \theta_{2}
$$

which, along with the Kähler metric (3.47) will determine a background similar to the one studied by Klebanov and Strassler in [1].

Similarly, we can determine the RR background fields. The RR fields are the axion $\tilde{\phi}$, the RR three form and the five form field strength. It is easy to see that

$$
\phi=0, \quad \tilde{\phi}=0 .
$$


The vanishing of $\tilde{\phi}$ is a consequence of mirror symmetry. To see that the dilaton also vanishes we have to consider the limit (2.6), where, without loss of generality we impose the following value for the expectation values of $\alpha$ and $\phi_{b}$ ( $\phi_{b}$ is the type IIA dilaton before the geometric transition):

$$
\langle\alpha\rangle=\left(64 e^{2\left\langle\phi_{b}\right\rangle}\right)^{-\frac{1}{3}}
$$

In fact, $\left\langle\phi_{b}\right\rangle$ is close to zero [15] and therefore (3.60) fixes the expectation value of $\alpha$ to a positive integer.

$B_{R R}$ can now easily be determined using mirror symmetry. From the exact supergravity background on the type IIA side we see that the gauge field has two components: along the $x$ and $\theta_{1}$ directions (2.12). In the $\mathrm{M}$-theory analysis of [15] this choice of gauge field was the simplest one. Of course, we could also consider other components, like $y$ and $\theta_{2}$. With this choice $(2.12)$, the background $B_{R R}$ field (in new coordinates) becomes

$$
B_{R R}=-2 A d \widehat{y} \wedge d \widehat{z}-\frac{2 e^{2 \phi} A^{2} \sqrt{\alpha}}{\epsilon C_{1}}\left(d \widehat{y}-\frac{e^{2 \phi} A B}{C_{1}} d \widehat{x}\right) \wedge d \theta_{1} .
$$

This can be compared with [1]. It is also easy to see that the five form background contains a factor of $b_{x \theta_{1}}$ or $b_{y \theta_{2}}$. This is crucial for the type IIB Bianchi identity to work out correctly.

Before we end this section, let us summarize the full type IIB background that we got by making a mirror transformation on the type IIA background:

$$
\begin{array}{r}
d s^{2}=h_{1}\left[d \widehat{z}+a_{1} d x+a_{2} d y\right]^{2}+h_{2}\left[d y^{2}+d \theta_{2}^{2}\right]+h_{4}\left[d x^{2}+h_{3} d \theta_{1}^{2}\right]+ \\
+h_{5} \sin \psi\left[d x d \theta_{2}+d y d \theta_{1}\right]+h_{5} \cos \psi\left[d \theta_{1} d \theta_{2}-d x d y\right] \\
B_{N S}=b_{x \theta_{1}} d x \wedge d \theta_{1}+b_{y \theta_{2}} d y \wedge d \theta_{2}, B_{R R}=-2 A d y \wedge d z, \quad \phi=\tilde{\phi}=0
\end{array}
$$

where we have omitted the ${ }^{\widehat{ }}$ on the coordinates to avoid cluttering of formulae.

\section{Supergravity Analysis in Type I}

To determine the supergravity backgrounds in type I theory we need to reconsider the whole scenario again. First, the background that we gave in the type IIB theory (or even in type IIA) will never allow us to go to type I and correspondingly to the heterotic theory. This is because type II theories have larger supersymmetries and to obtain the chiral nature of type I we have to cut down half of the world sheet supersymmetries. 
One way to cut down supersymmetries is by introducing an orientifold operation $\Omega$. However, as is well known, the operation that we need to generate the desired action so that we get to the type $\mathrm{I} /$ heterotic theory, is not simply $\Omega$, but a more involved one given by

$$
\Omega \cdot(-1)^{F_{L}} \cdot \sigma,
$$

where $(-1)^{F_{L}}$ reverses the space-time fermion numbers and $\sigma$ is a pure orbifold action which reverses an even number of directions.

The type IIB background that we studied in the previous section does not have an orientifold action. But we know how to introduce such an action: by lifting the type IIB background to F-theory. As studied in detail by Sen, the F-theory lift of a type IIB background has an orientifold limit in the moduli space which is determined in terms of some number of $D 7$ branes and $O 7$ planes located on the six dimensional base [28]. The F-theory lift requires a four-fold which is a non-trivial $T^{2}$ fibration over a six-dimensional base. To study the type I theory both before and after geometric transition, we need the six-dimensional base to be close to a resolved conifold or a deformed conifold consider the resolved conifold base.

The non-trivial $T^{2}$ fibration over a six-dimensional base needs to degenerate over some points on the base. Since the $T^{2}$ can degenerate over an even dimensional space we have the following four possibilities

(a) The $T^{2}$ degenerates over the full six-dimensional base.

(b) The $T^{2}$ degenerates over a four-dimensional subspace of the six-dimensional base.

(c) The $T^{2}$ degenerates over a two-dimensional subspace of the six-dimensional base.

(d) The $T^{2}$ does not degenerate over the base at all.

Looking at the four choices, we can easily eliminate (a), (b) and (d). For the first case, if the $T^{2}$ degenerates over the full six-dimensional base, then the $\sigma$ action of (4.1) will act on the full resolved conifold. This would imply that we need six T-dualities to convert (4.1) to simply $\Omega$

$$
\Omega \cdot(-1)^{F_{L}} \cdot \sigma \stackrel{T_{6}}{\longrightarrow} \Omega
$$

7 Recall that, in general, the four-fold base is not a Calabi-Yau manifold. Therefore, when we say the base is a resolved (or deformed) conifold we mean some metric that is close to the resolved (or deformed) conifold metric. It will soon be clear from the analysis below, what this means exactly. For the time being we will continue calling the base a resolved (or deformed) conifold. 
i.e. six T-dualities to go to the type I theory. Looking at the resolved conifold metric, we see that we can only have three isometries (along the $X, Y$ and $Z$ directions) 8 . Therefore, the first possibility cannot take us to the type I theory and therefore also not to the heterotic theory. Similarly, the next choice of four T-dualities fails because we do not have a sufficient number of isometries.

The fourth choice allows fluxes and branes because of non-compactness. However, any number of T-dualities will only keep us in the type II theory and will never take us out of this cycle. In other words, we can never go to the type I or heterotic theories using the choice (d).

The final choice (c) where the fiber degenerates over a two-dimensional subspace of the base seems to suffice for our purpose. There is one subtlety, as there are three possibilities for the two-dimensional subspace with coordinates: $X Y, Y Z$ and $X Z$. The question is then which one to choose, as we have equal isometries along the $X, Y$ and $Z$ directions. The answer to this question is found by looking at the base of the fourfold after the geometric transition, which changes from a resolved conifold to a deformed conifold, as described in [23]. The deformed conifold base does not have three isometries because it only has isometries along the $X$ and $Y$ directions. This would mean that if we choose the two-dimensional base to be along the $X$ and $Y$ directions then (both before and after the geometric transition) two T-dualities will take us to the type I theory (and then to heterotic). In this way we can uniquely fix the four-fold in F-theory to be a $T^{2}$ fibration over a two-dimensional base with coordinates $X$ and $Y$. In fact, the fiber torus will degenerate 24 times over the base $X, Y$ making this a $K 3$ manifold. This chain of arguments will allow us to determine the metric for the four-fold. Our first guess would be that the four-fold is nothing but a non-trivial product of a $K 3$ manifold and a four-cycle which we can specify precisely. The four-cycle, which we shall henceforth call $S_{4}$, will be different before and after the geometric transition. The metric for $S_{4}$ can be easily identified. Before the geometric transition the metric can be written as

$$
d s_{S_{4 r}}^{2}=\gamma^{\prime} \sqrt{h} d r^{2}+\left(d z+\Delta_{1} \cot \theta_{1} d x+\Delta_{2} \cot \theta_{2} d y\right)^{2}+\frac{\gamma \sqrt{h}}{4} d \theta_{1}^{2}+\frac{\left(\gamma+4 a^{2}\right) \sqrt{h}}{4} d \theta_{2}^{2},
$$

8 We will use the upper case letters to denote the coordinates, and lower case letters to write the metric components unless mentioned otherwise. 
where $\gamma, \gamma^{\prime}$ are defined in [20], [15]. The function $h$ is the overall warp factor coming from the back-reaction of the branes and fluxes on the geometry. Similarly, the metric of $S_{4}$ after the geometric transition is given by

$$
\begin{aligned}
d s_{S_{4 d}}^{2}= & h_{1}\left[d z+\widehat{\Delta}_{1} \cot \theta_{1} d x+\widehat{\Delta}_{2} \cot \theta_{2} d y\right]^{2}+h_{2} d \theta_{2}^{2}+h_{4} h_{3} d \theta_{1}^{2}+ \\
& +h^{1 / 2} \gamma^{\prime} d r^{2}+h_{5} \sin \psi\left[d x d \theta_{2}+d y d \theta_{1}\right]+h_{5} \cos \psi\left[d \theta_{1} d \theta_{2}-d x d y\right]
\end{aligned}
$$

where $h_{i}$ was already defined in (3.51). The other new coefficients $\widehat{\Delta}_{i}$ are defined as $\widehat{\Delta}_{1}=-\alpha_{0} e^{2 \phi} D \Delta_{1}$ and $\widehat{\Delta}_{2}=-\alpha_{0} e^{2 \phi} C \Delta_{2}$.

Looking at the metric of $S_{4 r}$ we see that it is of the form $S_{3} \times S^{1}$, where $S_{3}$ has coordinates $\left(\theta_{1}, \theta_{2}, r\right)$ and $S^{1}$ has coordinate $z$. Naively, one is led to conclude that the F-theory four-fold should be of the form $S_{3} \times S^{1} \otimes K 3$ with a metric $d s^{2}=d s_{S^{1}}^{2}+d s_{K 3}^{2}+d s_{S_{3}}^{2}$, where $\otimes$ denotes the non-trivial $S^{1}$ fibration over the $P^{1}$ base of $K 3$ with coordinates $X, Y$. The full metric of this system has also been worked out earlier in [48, [30, [23]. Unfortunately the situation is much more involved here and this naive choice of metric does not quite suffice for our case, as we shall explain below.

\subsection{Background before geometric transition}

As we discussed above, for the type I picture, we have to go to the orientifold corner of the F-theory moduli space. This has been discussed by [28], so we will be brief. Another relevant reference is 49 , that discusses the $\mathrm{F}$-theory lift of the Klebanov-Strassler solution. However, in none of the above references the metric for the orientifold corner of the moduli space has been computed. One might naively think that we can write the metric as mentioned above, but due to the $Z_{2}$ action this is a little subtle. The $Z_{2}$ action arises from the Weierstrass equation

$$
y^{2}=x^{3}+x f(u)+g(u)
$$

where $u$ is the coordinate of the base $\mathcal{M}$ and $f, g$ are some specific polynomials given in [28]. It gives rise to a type IIB compactification on $\mathcal{M}$ modded out by $Z_{2} \equiv \Omega \cdot(-1)^{F_{L}} \cdot \sigma$, where $\sigma$ is a pure orbifold operation

$$
\sigma: X \rightarrow-X, \quad Y \rightarrow-Y
$$

Now looking at (4.3) we see that the metric does not possess this symmetry. In fact, it is broken by the components $j_{x z}$ and $j_{y z}$. Furthermore, the existence of these components 
is already problematic if we want to go to the type I theory. After two T-dualities these components will transform into $B_{N S}$ fields and we know that the type I theory should not have $B_{N S}$ fields!

A way out of this problem is to untwist the $d z$ fibration. This can be achieved by going to the double cover, where we write the metric along the $z$ direction simply as $d z^{2}$. Recall that the twist of the $d z$ direction originates from having two Taub-NUT spaces. A T-dual version gives us two NS5 branes related to this [50], [51]. Alternatively the two Taub-NUT spaces become two intersecting $D 6$ branes in M-theory [52].

To obtain a metric with the required isometries that is also invariant under (4.6), we first remove the wrapped $D 5$ branes. This can be achieved by setting the harmonic function $h=1$. The metric of the resolved conifold after removing the wrapped $D 5$ branes becomes

$$
d s^{2}=d s_{x y}^{2}+d s_{Z}^{2}+d s_{S_{3}}^{2},
$$

with the notation used in earlier sections and in 15. We will keep the metric along the $X, Y$ and $Z$ directions as

$$
\begin{aligned}
& d s_{x y}^{2}=\left(1+A^{2}\right) d x^{2}+\left(1+B^{2}\right) d y^{2}+2 A B d x d y, \\
& d s_{Z}^{2}=d z^{2}+2 A d x d z+2 B d y d z,
\end{aligned}
$$

where $A, B$ are defined as before with the restriction $h=1$. From the above metric we see that the problematic components are $d x d z$ and $d y d z$ which are not invariant under (4.6). The metric along the $S_{3}$ direction can be written as

$$
d s_{S^{3}}^{2}=\gamma^{\prime} d r^{2}+\frac{\gamma}{4} d \theta_{1}^{2}+\frac{\gamma+4 a^{2}}{4} d \theta_{2}^{2}
$$

which is the same as before except that we have set $h=1$.

As discussed, the above metric (which is simply the resolved conifold metric without wrapped five branes) cannot suffice. We are looking for a metric in type IIB that has the following properties:

(a) It should be invariant under (4.6).

(b) It should preserve some number of supersymmetries (i.e. $\mathcal{N}=1$ ).

(c) It should be close to the original type IIB metric in terms of its form.

(d) It should allow wrapped $D 5$ branes along with some number of $D 7$ branes and $O 7$ planes, and 
(e) After two T-dualities, it should look close to the one obtained from T-dualizing the resolved conifold.

Let us start with (a) and (c). A generic metric that is invariant under (4.6) can be written in terms of two complex tori with complex coordinates $\chi_{1}$ and $\chi_{2}$ in the following way:

$$
d s^{2}=d_{1}\left|d \chi_{1}\right|^{2}+d_{2}\left|d \chi_{2}\right|^{2}+d_{3} d z^{2}+d_{4} d r^{2}
$$

where $d_{i}$ are some numerical coefficients that could in general be functions of the radial and angular coordinates, i.e.

$$
d_{i} \equiv d_{i}\left(\theta_{1}, \theta_{2}, r\right)
$$

which is motivated from the fact that the metric should have the required isometries along $X, Y$ and $Z$ directions. The complex coordinates $\chi_{i}$ above are defined in the following way:

$$
d \chi_{1}=d x+\tau_{1} d y, \quad d \chi_{2}=d \theta_{1}+\tau_{2} d \theta_{2}
$$

where $\tau_{i}$ are the complex structures of the given tori.

Expanding the above metric and keeping $\operatorname{Re} \tau_{2}=0$, we see that the components can be written as

$$
d s^{2}=d_{1}\left(d x^{2}+\left|\tau_{1}\right|^{2} d y^{2}+2 \operatorname{Re} \tau_{1} d x d y\right)+d_{2}\left(d \theta_{1}^{2}+\left|\tau_{2}\right|^{2} d \theta_{2}^{2}\right)+d_{3} d z^{2}+d_{4} d r^{2}
$$

We make the following observations regarding various issues here:

- The non-trivial coefficients $d_{i}$ cannot be arbitrary as the fourfold (which is a $T^{2}$ fibration) over the base (4.10) has to be a Ricci-flat Kähler manifold i.e. a CY fourfold. In the orientifold limit, the fourfold is $T^{4} / \mathcal{I}_{4} \times S_{4}$ where $T^{4} / \mathcal{I}_{4}$ is the orbifold limit of $K 3^{9}$ and $S_{4}$ is a four-cycle parameterized by $\theta_{1}, \theta_{2}, r, z$.

- There are three regions of interest here: (1) When we are at the orientifold point. The predicted metric at that point is the above metric (4.10). We expect the background axion-dilaton $(\tilde{\phi}, \phi)$ to be exactly cancelled in this background. (2) When we perturb one of the $D 7$ branes away from the $O 7$ plane. This creates a non-trivial axion-dilaton in the background. The metric is then calculated from the Seiberg-Witten curve. (3) When we take the axion-dilaton far away so that there would be a local region where the expectation

9 In fact it is a $T^{2}$ fibration over $T^{2} / \mathcal{I}_{2}$ base. As the reader can easily verify, this $T^{2} / \mathcal{I}_{2}$ is precisely a base with four fixed points [28], [53]. 
values of the axion-dilaton would be zero. In this paper we will only discuss (1) and (3), and leave (2) for future work.

- The full non-perturbative correction to this model can be worked out using the analysis of [28], [54. This non-perturbative correction arises when we move the $D 7$ branes away from the orientifold seven planes. This is very well known, so we will not repeat the discussion. In the end, this would convert the $T^{2} / \mathcal{I}_{2}$ parameterized by $X, Y$ to a $P^{1}$ with 24 singularities. These 24 singularities are the 24 zeroes of the discriminant of the Weierstrass equation (4.5).

Let us concentrate on the region when we have moved the seven branes far away. The generic metric along the $X, Y$ direction will be a $P^{1}$ with fixed points. If we call the $X, Y$ plane the $u$-plane 10 , then the metric of the $P^{1}$ will be

$$
d s^{2}=C \sum_{i} \frac{d u d \bar{u}}{\left|u-u_{i}\right|^{\frac{1}{2}}}
$$

where $u \equiv x+i y$ and $u_{i}$ are the points where the seven branes are located and $C$ is an appropriate constant. Consider now the case when we keep all the seven branes far away. In terms of the metric (4.13) this will imply:

$$
d s^{2}=C_{u_{i} \rightarrow \infty}\left(\sum_{i} \frac{d u d \bar{u}}{\left|u-u_{i}\right|^{\frac{1}{2}}}\right) \rightarrow \sum_{i} \frac{d u d \bar{u}}{\left|u_{i}\right|^{\frac{1}{2}}}, \quad\langle\tilde{\phi}\rangle \approx 0, \quad\langle\phi\rangle \approx 0 .
$$

This means there is a region in our space where we could see the two cycle of the resolved conifold on which we wrap $D 5$ branes. Since in this limit the metric along $X, Y$ directions is approximately flat, the two cycle is given by $\left(y, \theta_{2}\right)$ which is precisely the two cycle that we encountered in the resolved conifold setting. The metric of this region cannot be a Calabi-Yau due to back reactions from the seven branes. These back reactions can be calculated using an analysis similar to the one presented in [56], [57].

So far we discussed the situation when we put the seven branes far away. Let us come back to the case when we are at the orientifold point. The generic analysis using non trivial values for the $d_{i}$ in (4.10) is very involved. So, as a first approximation we will take all the $d_{i}$ to be constants. These constants are not arbitrary, and their choice will be motivated by the approximation that we used in (4.14), namely deforming the orientifold picture by moving the seven branes, so that the metric comes close to resembling a resolved conifold.

10 This is the same $u$-plane as that of Seiberg-Witten theory [55]. 
As we discussed in (4.14), we can identify the two cycles of the original resolved conifold. This is what we meant earlier by being close to the resolved conifold.

To identify various values here, we will use the approximation that we took in [15], namely, we set the radial coordinate to a fixed value. Thus, $\gamma$ etc. will be defined w.r.t. that value. We would like to remind the reader that this is just to simplify the ensuing analysis. A more detailed analysis can indeed be performed, but we will not do so here. Let us now define the generic complex structure of the two tori as:

$$
\tau_{1}=\frac{1}{1+A_{1}^{2}}\left[c A_{1} B_{1}+i \sqrt{\langle\alpha\rangle^{-1}+\left(1-c^{2}\right) A_{1}^{2} B_{1}^{2}}\right], \quad \tau_{2}=i \sqrt{1+\frac{4 a^{2}}{\gamma}}
$$

where $A_{1}, B_{1}$ are measured w.r.t. some average values of $\theta_{1}$ and $\theta_{2}$, respectively, as we had mentioned earlier in section 2 and also in [15]. We have also introduced an integer $c$ that can take values 1 or 0 . The precise value of $c$ will be determined by the choice of fluxes. We will soon evaluate this using the type IIB superpotential (1.1). For the choice of $c=1,0$, the complex structure of the two tori become

$$
\begin{array}{llll}
c=1: & \tau_{1}=\frac{1}{1+A_{1}^{2}}\left[A_{1} B_{1}+\frac{i}{\sqrt{\langle\alpha\rangle}}\right], & \tau_{2}=i \sqrt{1+\frac{4 a^{2}}{\gamma}} \\
c=0: & \tau_{1}=i \frac{\sqrt{\langle\alpha\rangle^{-1}+A_{1}^{2} B_{1}^{2}}}{1+A_{1}^{2}}, & \tau_{2}=i \sqrt{1+\frac{4 a^{2}}{\gamma} .}
\end{array}
$$

Furthermore, if we now allow the following values for the coefficients $d_{i}$ in $(4.10)$

$$
d_{1}=1+A_{1}^{2}, \quad d_{2}=\frac{\gamma}{4}, \quad d_{3}=1, \quad d_{4}=\gamma^{\prime}
$$

we see that the metric (4.10) comes very close to (4.7). In the limit (2.6) they will resemble exactly, but now without the problematic cross terms! In fact with the generic complex structure (4.15) the metric along the $x y$ directions take the form:

$$
d_{1}\left|d \chi_{1}\right|^{2}=\left(1+A_{1}^{2}\right) d x^{2}+\left(1+B_{1}^{2}\right) d y^{2}+2 c A_{1} B_{1} d x d y
$$

By construction, the metric preserves supersymmetry because it is written in terms of tori with non-trivial complex structure. To show that this metric preserves the precise amount of supersymmetry as that of the original resolved conifold, we have to project our space with $Z_{2}^{m}$ orbifold action, $m$ being some specific integer. Unfortunately, these $Z_{2}$ actions are not visible in the metric, so one cannot infer them from the above analysis. 
We have already taken care of the points (a), (b) and (c). Now let us deal with point (d). We require our metric (4.10) to allow wrapped D5 branes and also some number of $D 7$ branes and $O 7$ planes. A way to achieve this would be to lift the background to F-theory or M-theory. In M-theory the back-reaction of branes and fluxes can be easily evaluated along the lines proposed in [23]. The fluxes are of course the $B_{N S}$ fields and the $B_{R R}$ fields that form the sources of the wrapped $D 5$ branes. The final answer is gained by simply replacing the initial harmonic function $h$ with a different harmonic function $H$ that, from type IIB point of view, satisfies

$$
\square H=H_{N S} \wedge H_{R R}+\frac{\pi^{2} \alpha^{\prime 2}}{4} \sum_{i=1}^{24} b_{i} \operatorname{tr}\left(R_{i} \wedge R_{i}\right) \delta^{2}\left(z-z_{i}\right)
$$

where $i$ sums the number of branes and planes, each contributing a factor of $b_{i}$ amount of $\operatorname{tr}\left(R_{i} \wedge R_{i}\right)$. These $b_{i}$ 's have been worked out in [58]. The positions of these branes and planes on the $X, Y$ spaces are denoted by complex coordinates $z_{i}$. Wrapped $D 5$ branes, and D3 branes will have additional contributions to the warp factor. These details appeared in [30].

The direction $d r$ is non-compact and therefore the metric allows many wrapped $D 5$ branes with no restrictions from the anomaly condition [59]. The $2 d$ surface with metric $d s_{x y}^{2}$ will have points where the F-theory fiber degenerates, creating $D 7$ branes and $(p, q)$ seven branes. In special limits, these seven branes behave like non-dynamical 07 planes. Since $O$-planes are perturbative, these limits are at small type IIB coupling [28], [53]. The fact that this configuration of $D 5$ branes and seven branes preserves supersymmetry can be easily seen from the number of intersection points. In the figure below:

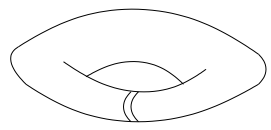

the $D 5$ branes wrap a torus along $\left(\theta_{2}, y\right)$ and extend along $x^{0,1,2,3}$. The seven branes are stretched along $\theta_{2}$ (given by the strip in the above figure) plus $\left(\theta_{1}, r, z\right)$ directions inside the manifold along with the usual $x^{0,1,2,3}$ directions. Such a configuration preserves supersymmetry. This way we take care of point $(d)$.

To further clarify the issue, we compare our results with the ones of [56], where D7 branes together with fractional D5 branes were considered. They dealt with an $\mathcal{N}=$ 2 theory as a $Z_{2}$ orbifold of the D3/D7 solution. The D3 branes were orthogonal to $R^{4} / Z_{2} \times R^{2}$ and the D7 branes were wrapped on the $R^{4} / Z_{2}$ and a point in $R^{2}$. At the 
orbifold point the singularity can be resolved by blowing up a $P^{1}$ cycle. If the $P^{1}$ cycle is of vanishing size and with a finite NS flux through it, then the D5 branes which are wrapped on $P^{1}$ are fractional D3 branes. A $\mathrm{T}$-dual on the angular direction of the vanishing $P^{1}$ cycle gives a type IIA brane configuration with NS branes and D4 branes 60. The $\mathcal{N}=2$ configuration of [56] can be rotated to one with $\mathcal{N}=1$ supersymmetry, where the $R^{4} / Z_{2}$ is replaced by a conifold singularity. In this case the fractional D3 branes are D5 branes wrapped on a vanishing $P^{1}$ cycle at the conifold point. The $\mathrm{T}$-dual is taken with respect to an $S^{1}$ in the base of the conifold and the $\mathrm{T}$-dual picture is an elliptic model with circular D4 brane [50] [51]. The worldvolume of the D7 branes contains the vanishing cycle.

It would then appear that the D7 branes and D5 branes have two common directions if the vanishing cycle is made finite. This would raise serious doubts as the configuration would be non-supersymmetric. But this is not an allowed process, the reason being discussed at length in [22]. We used the fact that in the conifold case, the distance between the NS branes in the T-dual picture is given by the flux of NS field through the vanishing 2-cycle. For the resolved conifold the distance between the NS branes in the T-dual picture is given by the size of the $P^{1}$. As these are two independent quantities, we cannot identify them. There is no continuous way to take the D7 on the conifold with fluxes to the D7 branes on the resolved conifold, as they have at least one different radial direction. Therefore we can have a D5-D7 intersection on one internal direction since the resolved conifold metric will allow this configuration 11. It would be very interesting to discuss in detail the supergravity solution by considering the back-reaction of the D7 branes, in the spirit of [56] (see also 61] for recent developments in this direction).

Before we discuss case (e), we should raise the important question whether the metric that we derived at the orientifold point (4.10) or (4.12) shows any geometric transition or not. Can we shrink the two cycle on which we have wrapped D5 branes and go to a background that has only fluxes with possible $D 7$ branes and $O 7$ planes? In other words, away from the orientifold point the type IIB metric with wrapped $D 5$ branes on a resolved conifold shows geometric transition. What happens at the orientifold point? Since, at the orientifold point we have removed the cross terms in the metric, it is a-priori not clear whether one can see the geometric transition or not. This might look a little disappointing, as we seem to lose the main reason of going to the type I scenario. One way out of this

11 We would like to thank Radu Roiban and Bogdan Florea for discussions on this and related issues. 
would be not to go to the orientifold point at all. But away from orientifold point we do not have any ways to go to type I!

This dilemma is resolved nicely by examining the metric in type I after two T-dualities on the metric (4.10). We find an interesting surprise here. Imagine we perform two Tdualities on the metric away from the orientifold point. Of course the final metric will remain in type IIB, but the two metrics 12 that we get by doing this look exactly the same up to a possible warp factor along the $d z$ direction! Thus, in this sense, the type I background that we will find after two T-dualities, will be dual to the type IIB background before geometric transition.

To confirm this claim, let us explicitly T-dualize the metric (4.10). Before we can do this we first require the following two steps:

- Insertion of the wrapped D5 branes along with the D7 and $O 7$ planes, and

- Specify the background $H_{N S}$ field at the orientifold point.

The first step is easy. We write the metric (4.10) but now with the harmonic function $H$ put in. This amounts to simply replacing $h$ in the original definition of $A_{1}, B_{1}$ by $H$, without any other changes.

For the second step, i.e. determining the value of the background $H_{N S}$, we recall that the only $H_{N S}$ field that is invariant under the orientifold projections should have one of its components 13 along the duality directions $X$ or $Y$. Although the original choice of $B_{N S}$ field (3.2) satisfies this condition, we will adopt a more generic ansatz. This is because the original choice (3.2) is suited for the specific metric that we had earlier. Since we are changing the metric by choosing all $d_{i}$ in (4.12) constant, we require a more general ansatz for the $B$ field. Let us therefore take

$$
B_{N S}=b_{x i} d x \wedge d \zeta^{i}+b_{y j} d y \wedge d \zeta^{j}
$$

where $\zeta^{i}=\left(\theta_{1}, \theta_{2}, z, r\right)$, generically. Since the components of $B$ field have one leg along the duality directions, we see that this would survive the orientifold action. We will also take both $b_{x i}$ and $b_{y j}$ to be functions of $\left(\theta_{1}, \theta_{2}, r\right) 14$.

12 One by T-dualizing (4.7) with $D 5, D 7$ and $(p, q)$ seven branes inserted in, and the other by T-dualizing (4.12) with $D 5, D 7$ branes and $O 7$ planes inserted in.

13 Recall that away from the orientifold point we can always excite other components of the $B$ field that do not have any legs along the duality directions. At the orientifold point these components are projected out.

14 The reason why the components are independent of functions along the $Z$ direction is because of mirror symmetry. 
The analysis including all the components along the $\left(\theta_{1}, \theta_{2}, z, r\right)$ directions is pretty involved, so we will simplify the analysis by first taking

$$
b_{x i} \equiv b_{x \theta_{1}}, \quad b_{y j} \equiv b_{y \theta_{2}}, \quad c=1 .
$$

These simplifying choices do not change any of the dualities that we will perform, as one can easily put back the other components to get the complete result. On the other hand these choices will be useful to see the fibration structure in the type I scenario in a simple way. The final metric after two T-dualities is similar to the one worked out in [30], [31], and is given by:

$$
\begin{aligned}
d s^{2}= & \frac{1}{g_{y y}}\left(d y+B_{\mu y} d x^{\mu}+B_{x y} d x+B_{z y} d z\right)^{2}-\frac{1}{g_{y y}}\left(g_{y \mu} d x^{\mu}+g_{y x} d x+g_{y z} d z\right)^{2} \\
& +g_{\mu \nu} d x^{\mu} d x^{\nu}+2 g_{x \nu} d x d x^{\nu}+2 g_{z \nu} d z d x^{\nu}+2 g_{x z} d x d z+g_{x x} d x^{2}+g_{z z} d z^{2}
\end{aligned}
$$

where $g_{m n}$ is the metric after one T-duality along $X$ direction:

$$
g_{m n}=\left(\begin{array}{ccccc}
\frac{1}{1+A_{1}^{2}} & 0 & 0 & -\frac{b_{x \theta_{1}}}{1+A_{1}^{2}} & 0 \\
0 & \frac{1}{\langle\alpha\rangle\left(1+A_{1}^{2}\right)} & 0 & 0 & 0 \\
0 & 0 & 1 & 0 & 0 \\
-\frac{b_{x \theta_{1}}}{1+A_{1}^{2}} & 0 & 0 & \frac{\gamma}{4}+\frac{b_{x \theta_{1}}^{2}}{1+A_{1}^{2}} & 0 \\
0 & 0 & 0 & 0 & \frac{\gamma+4 a^{2}}{4}
\end{array}\right) .
$$

Similarly, the $B_{N S}$ field after one T-duality appearing in (4.22) is given by:

$$
B_{m n}=\left(\begin{array}{ccccc}
0 & -\frac{A_{1} B_{1}}{1+A_{1}^{2}} & 0 & 0 & 0 \\
\frac{A_{1} B_{1}}{1+A_{1}^{2}} & 0 & 0 & -\frac{b_{x \theta_{1}} A_{1} B_{1}}{1+A_{1}^{2}} & b_{y \theta_{2}} \\
0 & 0 & 0 & 0 & 0 \\
0 & \frac{b_{x \theta_{1} A_{1} B_{1}}}{1+A_{1}^{2}} & 0 & 0 & 0 \\
0 & -b_{y \theta_{2}} & 0 & 0 & 0
\end{array}\right)
$$

where the components of the matrices for the metric and the $B$ field follow the same order as used so far. The final metric in type I is now found to be:

$$
\begin{gathered}
d s^{2}=\langle\alpha\rangle\left(1+A_{1}^{2}\right)\left(d y-b_{y \theta_{2}} d \theta_{2}\right)^{2}+\langle\alpha\rangle\left(1+B_{1}^{2}\right)\left(d x-b_{x \theta_{1}} d \theta_{1}\right)^{2}+\gamma^{\prime} \sqrt{H} d r^{2} \\
-2\langle\alpha\rangle A_{1} B_{1}\left(d x-b_{x \theta_{1}} d \theta_{1}\right)\left(d y-b_{y \theta_{2}} d \theta_{2}\right)+d z^{2}+d_{2}\left|d \chi_{2}\right|^{2}
\end{gathered}
$$


with vanishing $B_{N S}$. The above metric follows exactly the criteria laid down in [15], namely, whenever we see $d x$ and $d y$ we have to replace them with the $b$ dependent fibration $\left(d x-b_{x \theta_{1}} d \theta_{1}\right)$ and $\left(d y-b_{y \theta_{2}} d \theta_{2}\right)$, respectively. The only other change is the warp factors in front of the metric components. By construction this metric can be easily shown to be non-Kähler. A similar fibration structure, leading to a non-Kähler metric, was proposed earlier in a series of papers [30], [31], [32], 333](see also [62]).

Let us now show that this metric is indeed dual to some type IIB metric. We will explicitly T-dualize the type IIB metric away from the orientifold point, i.e. our starting metric will be (4.7). Here we use the approximation (4.14), i.e. the seven branes are far away and therefore the base metric can be approximated by a resolved conifold. The $B_{N S}$ field remains the same with components $b_{x \theta_{1}}$ and $b_{y \theta_{2}}$. As we have seen, these components not only survive the orientifold projections, but are also solution to the equation of motion. After two T-dualities the metric will be similar to (4.22), but we will remain in type IIB theory. The values of $g_{m n}$ appearing in (4.22) are:

$$
g_{m n}=\left(\begin{array}{ccccc}
\frac{1}{1+A^{2}} & 0 & 0 & -\frac{b_{x \theta_{1}}}{1+A^{2}} & 0 \\
0 & \frac{1}{\alpha\left(1+A^{2}\right)} & \frac{B}{1+A^{2}} & 0 & 0 \\
0 & \frac{B}{1+A^{2}} & \frac{1}{1+A^{2}} & 0 & 0 \\
-\frac{b_{x \theta_{1}}}{1+A^{2}} & 0 & 0 & \frac{\gamma}{4}+\frac{b_{x \theta_{1}}^{2}}{1+A^{2}} & 0 \\
0 & 0 & 0 & 0 & \frac{\gamma+4 a^{2}}{4}
\end{array}\right)
$$

Comparing with (4.23) we see that there are some specific changes. First, the $d z^{2}$ component is now no longer 1 but is more complicated. We have also developed a new component along the $d y d z$ direction, that was absent in (4.23). Furthermore, all the other components have remained the same, but are not measured w.r.t. the expectation values any more, they will coincide with the approximation (2.6). On the other hand, the $B_{N S}$ field turns out to be:

$$
B_{m n}=\left(\begin{array}{ccccc}
0 & -\frac{A B}{1+A^{2}} & -\frac{A}{1+A^{2}} & 0 & 0 \\
\frac{A B}{1+A^{2}} & 0 & 0 & -\frac{b_{x \theta_{1} A B}}{1+A^{2}} & b_{y \theta_{2}} \\
\frac{A}{1+A^{2}} & 0 & 0 & -\frac{b_{x \theta_{1}} A}{1+A^{2}} & 0 \\
0 & \frac{b_{x \theta_{1}} A B}{1+A^{2}} & \frac{b_{x \theta_{1}} A}{1+A^{2}} & 0 & 0 \\
0 & -b_{y \theta_{2}} & 0 & 0 & 0
\end{array}\right)
$$

Again, comparing with (4.24), we see that we have developed new components along $d z \wedge d \theta_{1}$ and $d x \wedge d z$ directions. These new components are related to the cross terms in 
the type IIB metric away from the orientifold point. The final metric in type IIB after two $\mathrm{T}$-dualities therefore will take the following form:

$$
\begin{aligned}
d s^{2}= & \alpha\left(1+A^{2}\right)\left(d y-b_{y \theta_{2}} d \theta_{2}\right)^{2}+\alpha\left(1+B_{1}^{2}\right)\left(d x-b_{x \theta_{1}} d \theta_{1}\right)^{2}+\gamma^{\prime} \sqrt{H} d r^{2} \\
& -2 \alpha A B\left(d x-b_{x \theta_{1}} d \theta_{1}\right)\left(d y-b_{y \theta_{2}} d \theta_{2}\right)+\alpha d z^{2}+d_{2}\left|d \chi_{2}\right|^{2}
\end{aligned}
$$

with the corresponding $B_{N S}$ given by

$$
B_{N S}^{(2)}=-\alpha A\left(d x-b_{x \theta_{1}} d \theta_{1}\right) \wedge d z-\alpha B\left(d y-b_{y \theta_{2}} d \theta_{2}\right) \wedge d z
$$

Compare this metric with the one that we got for type I theory (4.25). We see that if we impose the limit (2.6), these two metrics are exactly the same up to one possible warp factor for the $d z^{2}$ directions. For the metric (4.25), the $d z^{2}$ term comes with coefficient 1 , whereas in (4.28) the $d z^{2}$ term comes with coefficient $\langle\alpha\rangle$ when we use (2.6). This if we redefine the $z$ direction in $(4.25)$ as

$$
z=\sqrt{\langle\alpha\rangle} z^{\prime}
$$

then the two metrics will coincide exactly. The metric in type IIB (4.28) shows geometric transition because it is $\mathrm{T}$-dual to the original type IIB metric that showed geometric transition. Using this analogy, we see that the type I metric will form the precise dual to the type IIB metric and therefore this will provide the geometric transition dual to type IIB in the type I theory.

In terms of our previous discussion, after the two $\mathrm{T}$-dualities the three regions with D7 and O7 overlap such that it is natural to obtain an identical metric after taking 2 $\mathrm{T}$-dualities for each of them. In type I there is no distinction between regions which are close or far away from the orientifold plane as the orientifold plane is an O9 plane and there is no extra direction to separate from it.

At this point we have to study what this background represent in the type I theory. Since the background originates directly from type IIB via T-dualities, it should be related to wrapped D5 branes on this non-Kähler background. In fact, as we will discuss soon, under an S-duality this will be transported to heterotic theory on a non-Kähler background with NS5 branes. The NS5 branes are the sources of torsion in this scenario. This fits exactly with the picture developed in 63]. We will discuss more on this connection in section 5. But first we need the other fields, i.e. the RR three form and the dilaton for the type I background. 
To get the $H_{R R}$ for the type I we cannot simply T-dualize $H_{R R}$ of type IIB (3.3), because $H_{R R}$ of type IIB is in fact not invariant under the orientifold action. The problematic components are:

$$
c_{2} \cot \widehat{\theta}_{1} d x \wedge d \theta_{2} \wedge d y-c_{3} \cot \widehat{\theta}_{2} d y \wedge d \theta_{1} \wedge d x
$$

that pick up minus signs under the orientifold action. The coefficient $c_{2}, c_{3}$ are defined in [15], [20]. The only components that could remain invariant under the orientifold action will be the ones that have one leg along $X$ or $Y$ directions. Let us therefore use the following ansatz for the RR field in type IIB theory at the orientifold point:

$$
\begin{aligned}
& H_{R R}^{b}= \mathcal{H}_{y k l}^{b} d y \wedge d \zeta^{k} \wedge d \zeta^{l}+\mathcal{H}_{x i j}^{b} d x \wedge d \zeta^{i} \wedge d \zeta^{j} \\
&= \mathcal{H}_{y z \theta_{2}}^{b} d y \wedge d z \wedge d \theta_{2}+\mathcal{H}_{x z \theta_{1}}^{b} d x \wedge d z \wedge d \theta_{1}+ \\
& \quad+\mathcal{H}_{x z r}^{b} d x \wedge d z \wedge d r+\mathcal{H}_{y z r}^{b} d y \wedge d z \wedge d r
\end{aligned}
$$

along with the $B_{N S}$ field given by (4.20)15. The axion-dilaton is zero because at the orientifold point the seven brane charges are cancelled exactly. Along with the metric (4.10), (4.16) and (4.17), the above set of background will provide the complete solution of the type IIB system at the orientifold point.

Before we move ahead, we need to see whether the choices of $H_{R R}^{b}$ and $H_{N S}$ are compatible with the metric that we have at the orientifold point (4.10). A careful look at the metric tells us that the metric is actually a toroidal orbifold with non-trivial complex structures. This situation is now similar to the one that was seen earlier in [31], namely, a toroidal orbifold compactification of type IIB theory with fluxes. As in [31], the fluxes will fix the complex structures of the type IIB manifold (4.10) at the orientifold point. One choice of $H_{N S}$ and $H_{R R}^{b}$ fluxes that would do the job are constant fluxes (this doesn't mean that the $B_{N S}$ and $B_{R R}$ are constants). For such fluxes, there is a constraint coming from the choice of the components:

$$
B_{[x m}^{N S} B_{n y]}^{R R}=0
$$

which was shown in [31], [33]. This constraint has implications when we determine the type I RR field.

15 Observe that we have chosen only few components of the three-form field. This is for simplicity. We can always choose all the twelve components. None of the analysis will be affected by this choice. 
Performing two T-dualities on this background will determine the type I theory for us. We have already used this to get the metric. For the generic choice of $B_{N S}$ field, the fibration structure of the type I metric will simply change to

$$
\left(d x-b_{x \theta_{1}} d \theta_{1}\right) \rightarrow\left(d x-b_{x i} d \zeta^{i}\right), \quad\left(d y-b_{y \theta_{2}} d \theta_{2}\right) \rightarrow\left(d y-b_{y j} d \zeta^{j}\right) .
$$

The question now is whether there are other changes in the metric when we choose constant background fluxes. It turns out when the background fluxes are arbitrary and not constants, the value of $c$ is typically 1 . For constant background fluxes, as the one chosen in [31], [30] the value of $c$ turns out to be

$$
c=0 \text {. }
$$

This can be easily shown by minimising the type IIB superpotential (1.1). It turns out that (4.34) and (4.35) are only changes that we have to do to the metric.

Let us now evaluate the RR fields of type I. Something interesting happens now. When we perform two T-dualities on the type IIB background, the components of type I RR field that are orthogonal to the duality directions $X, Y$ take the following form 16 :

$$
B_{m n}^{I}=a_{0} D_{x y m n}^{+}+a_{1} B_{[x m}^{N S} B_{n y]}^{R R}+a_{2} B_{x[m}^{N S} B_{n] x}^{R R}+a_{3} B_{y[m}^{N S} B_{n] y}^{N S}
$$

with $D^{+}$being the four-form that has both legs along the duality directions. This vanishes trivially because in the presence of NS and RR three-forms we do not require the five form to be self-dual. The $a_{i}$ now take the following values:

$$
a_{0}=1, \quad a_{1}=6, \quad a_{2}=\frac{c}{1+A_{1}^{2}}, \quad a_{3}=-2 \tilde{\phi}
$$

where $\tilde{\phi}$ is the type IIB axion background. Imposing the constraints (4.33) and (4.35) we see that the type I three-form fields that do not have any legs 17 along the duality

16 See [31] for a derivation of this.

17 The type I $B$ field that has both legs along the duality directions also vanish because for this $a_{0}=a_{1}=a_{2}=0, \quad a_{3}=-\tilde{\phi}$. 
directions vanish! Therefore performing two T-dualities on (4.32) (and the coupling) gives the following type I RR field (and coupling):

$$
\begin{aligned}
H_{R R}^{I}= & \mathcal{H}_{x z \theta_{1}}^{b} d y \wedge d z \wedge d \theta_{2}-\mathcal{H}_{y z \theta_{2}}^{b} d x \wedge d z \wedge d \theta_{1}+ \\
& +\mathcal{H}_{x z r}^{b} d y \wedge d z \wedge d r-\mathcal{H}_{y z r}^{b} d x \wedge d z \wedge d r \\
g^{I}= & \sqrt{\langle\alpha\rangle}
\end{aligned}
$$

which along with the metric (4.25) will determine the type I dual background before geometric transition in type IIB theory.

There are several issues that need to be mentioned at this point. First, observe that the dilaton in type I theory no longer vanishes, even though the corresponding type IIB theory has a vanishing dilaton-axion. Second, we still have to determine the background values of $H_{R R}^{b}$. We shall determine them below and compare the result using torsional constraints [44], [36] later when we go to the heterotic theory. Third, in determining the $H_{R R}^{I}$ we see the $d x, d y$ terms do not have the expected fibration structures that the type I metric possesses. This phenomenon was also observed in [31], [30] where the metric showed the fibration structure but the $B$ field did not. The reason for this is simple. Any $z \theta_{i}-\operatorname{cross}$ terms in the $B$ field would in fact come from a term that is proportional to (4.33) as we saw in (4.36) (see also [31] for a derivation of this). Since this term is identically zero, the $B_{R R}$ lacks any fibration structure. This reasoning would remain true for completely generic $B_{N S}$ and $B_{R R}$ E: (4.33) would serve to eliminate all components that do not have one leg along $X$ or $Y$, ergo to forbid any fibration structure. Fourth, the background that we determined for type $\mathrm{I}$ is in fact incomplete, because we have not specified the gauge bundle yet. The gauge bundle has to satisfy the usual Donaldson-Uhlenbeck-Yau (DUY) equation

$$
g^{a \bar{b}} F_{a \bar{b}}^{i}=0
$$

where $g$ is the type I metric (4.25) written in terms of complex coordinates, and $i$ counts the number of D9 branes along with the Wilson lines on it; along with another constraint on $\operatorname{tr} F^{i} \wedge F^{i}$ :

$$
\operatorname{tr} F^{i} \wedge F^{i}=\operatorname{tr} R^{i} \wedge R^{i}-i \partial \bar{\partial} J
$$

18 Note, that $B_{N S}$ and $B_{R R}$ are still constrained by the orientifold action, so the most generic case would only allow $x z, x \theta_{1}, x \theta_{2}$ and $x r$ components (plus $x \leftrightarrow y$ ) 
which was first discussed in some details in [33]. The solutions to these two equations are rather non-trivial, so we will not address them here. Furthermore, the fact that we can write the DUY equations in terms of $a, \bar{b}$ means that the complex structure in type I theory is integrable even though the manifold is non-Kähler. We already encountered such kind of manifolds in [30], [31], [32], [33]. The difference is that those manifolds were compact. Here we find new complex non-Kähler manifolds that are non-compact. On the other hand, the non-Kähler manifolds encountered in [15] for the type IIA theory were non-complex and non-Kähler. It is interesting to see that as we move down the duality chain we go from non-complex, non-Kähler in type IIA to complex Kähler in type IIB and then to complex non-Kähler in type I (and also in heterotic)!

To determine the background $B_{R R}^{b}$ we can use the type IIB supergravity equation of motion. We already know the background $B_{N S}^{b}$ and the metric along with the axiondilaton. Therefore, the background supergravity equations are much simpler here. In fact, they become even simpler if we use the supersymmetry variation equations. The second order differential equations for the $B_{R R}^{b}$ become first order equations:

$$
H_{R R}^{b} \equiv \mathcal{H}^{b}=* H_{N S}^{b}
$$

as conjectured first in [1], and later shown to occur from the primitivity equation 19 in M-theory [23]. Now using the $B_{N S}^{b}$ field and (4.41), we can determine the non-zero components of $H_{R R}$ as 20 .

$$
\begin{array}{ll}
\mathcal{H}_{r y z}^{b}=\alpha_{1} \partial_{\left[\theta_{2} b_{\left.x \theta_{1}\right]}\right]}, & \mathcal{H}_{\theta_{2} y z}^{b}=\alpha_{2} \partial_{[r} b_{\left.x \theta_{1}\right]} \\
\mathcal{H}_{\theta_{1} x z}^{b}=\alpha_{3} \partial_{[r} b_{\left.y \theta_{2}\right]}, & \mathcal{H}_{r x z}^{b}=\alpha_{4} \partial_{\left[\theta_{1}\right.} b_{\left.y \theta_{2}\right]}
\end{array}
$$

where the coefficients $\alpha_{i}$ are determined from the Hodge star and the epsilon tensor that appears in (4.41).

19 The primitivity equation basically tells us what choices of fluxes on a given fourfold preserve supersymmetry 48.

20 Again, the generic choice will involve twelve equations. We will however continue using these components only. 


\subsection{Background after geometric transition}

Having given the background before geometric transition, we now determine the corresponding metric after geometric transition in type I theory. To do so we have to go through the details that we discussed for the case before geometric transition again. Some preliminary analysis has been done earlier in [49] and [23] where fourfolds with bases having a conifold singularity were discussed (see also [64]). In [49] the base of the fourfold was shown to be a hypersurface given by a quartic equation in $P^{4}$ :

$$
z_{5}^{2}\left(\sum_{i=1}^{4} z_{i}^{2}\right)-t^{2} z_{5}^{4}+\sum_{i=1}^{4} z_{i}^{4}=0
$$

where $z_{i}$ are the homogeneous coordinates of $P^{4}$ and $t$ is a real parameter. In [23], a similar but compact model was constructed with Euler number $\chi=19728$. The 49 model, that is non-compact has Euler number $\chi=1728$.

The fourfold can be constructed by a $T^{2}$ fibration over this hypersurface. At a generic point this allows $(p, q)$ seven branes. The above hypersurface can be analyzed at the locus where $z_{5}=1$. We see that (4.43) reduces over this locus to

$$
\sum_{i=1}^{4}\left(z_{i}^{4}+z_{i}^{2}\right)=t^{2}
$$

which, when taking the real part of $z_{i}$, becomes the equation for an $S^{3}$ of a deformed conifold. This is again the limit with the seven branes far away. The metric away from the

orientifold plane can therefore be approximated by the one given in (3.47), with the warp factors $h_{i}$ as defined in (3.51). At the orientifold point, this metric clearly cannot suffice, because of the non-zero $d x d z, d y d z, d x d \theta_{2}$ and $d y d \theta_{1}$ components. The components $d x d \theta_{2}$ and $d y d \theta_{1}$ can be eliminated by the usual trick that we applied earlier, namely using the transformation equation (4.33) of [15]. In this way the starting type IIB background away from the orientifold point (and with $h$ replaced by $H$ in the warp factors $h_{i}$ ) can be approximated as:

$$
\begin{aligned}
d s^{2}= & h_{1}\left[d z+a_{1} d x+a_{2} d y\right]^{2}+h_{2}\left[d y^{2}+d \theta_{2}^{2}\right]+h_{4}\left[d x^{2}+h_{3} d \theta_{1}^{2}\right] \\
& +h_{5}\left[d \theta_{1} d \theta_{2}-d x d y\right]+\gamma^{\prime} \sqrt{H} d r^{2} \\
B_{N S}= & b_{x \theta_{1}} d x \wedge d \theta_{1}+b_{y \theta_{2}} d y \wedge d \theta_{2}
\end{aligned}
$$


which is a Kähler background and is in fact precisely the Klebanov-Strassler background under the transformation (4.33) of [15] (and with the $d z$ direction delocalized). We have also used $x, y$ to denote the coordinates instead of $\widehat{x}, \widehat{y}$ to avoid clutter.

At the orientifold point we still cannot use the above metric because of the cross terms $d x d z$ and $d y d z$. The metric that we require would follow the criteria laid down earlier (before geometric transition). The metric will again look like (4.10):

$$
d s^{2}=b_{1}\left|d \chi_{1}\right|^{2}+b_{2}\left|d \chi_{2}\right|^{2}+b_{3} d z^{2}+b_{4} d r^{2}
$$

but now the complex structures will be different. In fact, due to the relation (which is easy to see from $(3.51))$ :

$$
h_{5}=2 a_{1} a_{2} h_{1} \quad=>\operatorname{Re} \tau_{1}=0
$$

but $\operatorname{Re} \tau_{2} \neq 0$. This is opposite to what we had earlier: $\operatorname{Re} \tau_{1} \neq 0$ and $\operatorname{Re} \tau_{2}=0$ for generic $c$. This also means that (4.46) naturally has $c=0$.

At this point we see that the $b_{i}$ defined above are again functions of the radial and the angular coordinates i.e. $b_{i} \equiv b_{i}\left(\theta_{1}, \theta_{2}, r\right)$. The generic analysis with non-trivial functions of $b_{i}$ is complicated and therefore we will approximate these coefficients by constants. These constant values will be motivated by the fact that in some region, when we move the seven branes away, the space is given by the hypersurface (4.44) and therefore the metric will resemble (4.45). Now imposing the condition (2.6), and taking the same symbol $h_{i}$ to represent the expectation values of $h_{i}$ 's, we can write the complex structure of the two tori in (4.10) as:

$$
\tau_{1}=i \sqrt{\frac{h_{2}+a_{2}^{2} h_{1}}{h_{4}+a_{1}^{2} h_{1}}}, \quad \tau_{2}=\frac{1}{h_{3} h_{4}}\left[a_{1} a_{2} h_{1}+i \sqrt{h_{2} h_{3} h_{4}-a_{1}^{2} a_{2}^{2} h_{1}^{2}}\right] .
$$

The coefficients $b_{i}$ in $(4.46)$ will be given by:

$$
b_{1}=h_{4}+a_{1}^{2} h_{1}, \quad b_{2}=h_{3} h_{4}, \quad b_{3}=h_{1}, \quad b_{4}=\gamma^{\prime} \sqrt{H}
$$

where $H$ has already been derived earlier. The metric after two $\mathrm{T}$-dualities in type I will again have the same form as $(4.22)$, but now $g_{m n}$ will be given by the following matrix:

$$
g_{m n}=\left(\begin{array}{ccccc}
\frac{1}{h_{4}+a_{1}^{2} h_{1}} & 0 & 0 & -\frac{b_{x \theta_{1}}}{h_{4}+a_{1}^{2} h_{1}} & 0 \\
0 & h_{2}+a_{2}^{2} h_{1} & 0 & 0 & 0 \\
0 & 0 & h_{1} & 0 & 0 \\
\frac{-b_{x \theta_{1}}}{h_{4}+a_{1}^{2} h_{1}} & 0 & 0 & h_{3} h_{4}+\frac{b_{x \theta_{1}}^{2}}{h_{4}+a_{1}^{2} h_{1}} & h_{5} / 2 \\
0 & 0 & 0 & h_{5} / 2 & h_{2}
\end{array}\right)
$$


where $h_{i}$ are the expectation values of $h_{i}$, unless mentioned otherwise. Observe that we have taken the $B_{N S}$ field to be (4.20) again with the same simplifying choices that we made earlier. Observe also that the terms in the above matrix are different from the ones that we had in (4.23) or (4.26). This is expected from the choice of type IIB background (4.45) itself . On the other hand, the $B$ field appearing in (4.22) is much simpler than its cousin (4.24), and (4.27). In matrix form it is given by:

$$
B_{m n}=\left(\begin{array}{ccccc}
0 & 0 & 0 & 0 & 0 \\
0 & 0 & 0 & 0 & b_{y \theta_{2}} \\
0 & 0 & 0 & 0 & 0 \\
0 & 0 & 0 & 0 & 0 \\
0 & -b_{y \theta_{2}} & 0 & 0 & 0
\end{array}\right)
$$

where as we see, most of the components are zero. The fact that only $b_{y \theta_{2}}$ appears is clear, as the other component of the $B$ field $b_{x} \theta_{1}$ dissolves in the metric. Using these matrices, the final type I metric can be written in a concise way as:

$$
\begin{gathered}
d s^{2}=\frac{1}{h_{2}+a_{2}^{2} h_{1}}\left(d y-b_{y \theta_{2}} d \theta_{2}\right)^{2}+\frac{1}{h_{4}+a_{1}^{2} h_{1}}\left(d x-b_{x \theta_{1}} d \theta_{1}\right)^{2} \\
+h_{1} d z^{2}+h_{3} h_{4}\left|d \chi_{2}\right|^{2}+\gamma^{\prime} \sqrt{H} d r^{2}
\end{gathered}
$$

with, of course, vanishing $B_{N S}$.

To show that this metric is indeed the geometric transition dual of the type I metric in (4.25) we have to show that it is also in some sense dual to the IIB metric after geometric transition. So, we need to find the metric away from the orientifold point, after two T-dualities. The final metric is of course (4.22), but now $g_{m n}$ is given by:

$$
g_{m n}=\left(\begin{array}{ccccc}
\frac{1}{h_{4}+a_{1}^{2} h_{1}} & 0 & 0 & \frac{-b_{x \theta_{1}}}{h_{4}+a_{1}^{2} h_{1}} & 0 \\
0 & h_{2}+a_{2}^{2} h_{1} & a_{2} h_{1} & 0 & 0 \\
0 & a_{2} h_{1} & \frac{h_{1} h_{4}}{h_{4}+a_{1}^{2} h_{1}} & 0 & 0 \\
-\frac{b_{x \theta_{1}}}{h_{4}+a_{1}^{2} h_{1}} & 0 & 0 & h_{3} h_{4}+\frac{b_{x \theta_{1}}^{2}}{h_{4}+a_{1}^{2} h_{1}} & h_{5} / 2 \\
0 & 0 & 0 & h_{5} / 2 & h_{2}
\end{array}\right)
$$

where $h_{i}$ no longer represent the expectation values any more. Observe that we have, as before, developed non-zero cross terms $d y d z$. And similarly the $d z^{2}$ term has become 
more complicated. The $B$ field also develops more components from the ones that we had in $(4.51)$, and is now given by

$$
B_{m n}=\left(\begin{array}{ccccc}
0 & 0 & -\frac{a_{1} h_{1}}{h_{4}+a_{1}^{2} h_{1}} & 0 & 0 \\
0 & 0 & 0 & 0 & b_{y \theta_{2}} \\
\frac{a_{1} h_{1}}{h_{4}+a_{1}^{2} h_{1}} & 0 & 0 & \frac{b_{x \theta_{1}} a_{1} h_{1}}{h_{4}+a_{1}^{2} h_{1}} & 0 \\
0 & 0 & -\frac{b_{x \theta_{1}} a_{1} h_{1}}{h_{4}+a_{1}^{2} h_{1}} & 0 & 0 \\
0 & -b_{y \theta_{2}} & 0 & 0 & 0
\end{array}\right)
$$

where again $h_{i}$ are not the expectation values any more. With these, the final type IIB metric after two T-dualities will now be given by:

$$
\begin{gathered}
d s^{2}=\frac{1}{h_{2}+a_{2}^{2} h_{1}}\left(d y-b_{y \theta_{2}} d \theta_{2}\right)^{2}+\frac{1}{h_{4}+a_{1}^{2} h_{1}}\left(d x-b_{x \theta_{1}} d \theta_{1}\right)^{2}+\gamma^{\prime} \sqrt{H} d r^{2} \\
+h_{1}\left[\frac{h_{2} h_{4}-a_{1}^{2} a_{2}^{2} h_{1}^{2}}{\left(h_{4}+a_{1}^{2} h_{1}\right)\left(h_{2}+a_{2}^{2} h_{1}\right)}\right] d z^{2}+h_{3} h_{4}\left|d \chi_{2}\right|^{2} .
\end{gathered}
$$

Comparing (4.55) and (4.52) we see that imposing (2.6) the two metrics are exactly identical up to a possible warp factor for the $d z$ direction. Thus (4.52) will form the dual to type IIB theory after geometric transition.

We now want to consider the corresponding RR background and coupling in type I theory after geometric transition. For this again we have to look at the RR background in type IIB theory at the orientifold point, that is invariant under the orientifold action. Let us therefore make the following ansatz for the RR background in type IIB theory:

$$
\begin{aligned}
H_{R R}^{b}= & \tilde{\mathcal{H}}_{y z \theta_{2}}^{b} d y \wedge d z \wedge d \theta_{2}+\tilde{\mathcal{H}}_{x z \theta_{1}}^{b} d x \wedge d z \wedge d \theta_{1}+ \\
& \tilde{\mathcal{H}}_{x z r}^{b} d x \wedge d z \wedge d r+\tilde{\mathcal{H}}_{y z r}^{b} d y \wedge d z \wedge d r
\end{aligned}
$$

which is similar to the $H_{R R}^{b}$ choice that we made earlier. The values for $\tilde{H}^{b}$ will be given by an equation similar to $(4.42)$ with $\mathcal{H}^{b}$ replaced by $\tilde{\mathcal{H}}^{b}$ with no other changes. We will be able to compare this with another calculation later when we go to the heterotic side. The type I coupling and the RR form can now be determined as

$$
\begin{aligned}
H_{R R}^{I}= & \tilde{\mathcal{H}}_{x z \theta_{1}}^{b} d y \wedge d z \wedge d \theta_{2}-\tilde{\mathcal{H}}_{y z \theta_{2}}^{b} d x \wedge d z \wedge d \theta_{1}+ \\
& +\tilde{\mathcal{H}}_{x z r}^{b} d y \wedge d z \wedge d r-\tilde{\mathcal{H}}_{y z r}^{b} d x \wedge d z \wedge d r \\
g^{I}= & \left(h_{4}+a_{1}^{2} h_{1}\right)^{-1 / 2}\left(h_{2}+a_{2}^{2} h_{1}\right)^{-1 / 2}=\sqrt{C^{\prime} D^{\prime}}
\end{aligned}
$$


The coupling turns again out to be constant as $C^{\prime}$ and $D^{\prime}$ are to be understood as expectation values, i.e. $C$ and $D$ at fixed radius and angle. Thus, they will be independent of functions of the internal coordinates of the manifold. The complete background can be determined once we specify the solution to DUY equation (4.39) as before.

\section{Supergravity Analysis in Heterotic Theory}

Now that we have the type I background, it is time to find the corresponding heterotic background. This is of course straight forward, as the heterotic background is simply the S-dual of type I above. But there is more to it. Since the heterotic background comes from S-dualizing the type I background, it inherits the $B$ field of type I. This is now interesting because the $B$ field or equivalently the $H$ field will in fact make this a torsional background. Therefore we are in the realm of [44], [36], [32], [33] where a detailed study of such backgrounds was performed, albeit, for a compact space. Here we have new torsional backgrounds that are non-compact, non-Kähler but complex.

Having a complex non-Kähler background means that we can use the mathematical construction developed in [32], [65], [66], [13], [33]. Since all these details have been extensively described there, we will avoid discussing them here. Our description will therefore be the bare minimum necessary for exposing the subject.

From the detailed studies done in [32, [33] (see also [44]) it is clear that these torsional backgrounds allow two different choices of spin connections $\omega_{+}$and $\omega_{-}$, which are determined by $\pm \frac{1}{2} H$. The question now is to see whether an equivalent of standard embedding [67] is also allowed here. As discussed in [32] standard embedding here means the following equation:

$$
A=\omega_{+}
$$

where $A$ is the gauge bundle satisfying DUY equation (4.39). The fact that the other spin connection does not appear in this equation has been explained in [32]. Existence of this equation implies that the $(0,2)$ supersymmetric non-linear sigma model (see second reference of [44]) defined on this background satisfying the necessary conditions is finite to all orders2]. However, for the torsional background that was studied in [32], [33] and also the one that we are going to study here, the equation (5.1) is not allowed. For the compact case, this was argued in [32] (see also [69]). Allowing (5.1) would make the warp factor

21 To two loop orders it has been shown in 68 . 
trivial and therefore the manifold would become Kähler. We believe a similar argument will go through for the non-compact case, too. This does not imply that the $(0,2)$ sigma model is not finite here. The fact that now $d H \neq 0$ and the manifold is non-compact modify the argumentation. A full analysis of this has not been done yet and we hope to address it in the near future.

\subsection{Background before geometric transition}

After this general discussion, let us go back to the precise model in question. The supergravity background for the heterotic case can be determined by $\mathrm{S}$-dualizing the type I background. The metric for our space will be given as

$$
\begin{gathered}
d s^{2}=\sqrt{\langle\alpha\rangle}\left(1+A_{1}^{2}\right)\left(d y-b_{y j} d \zeta^{j}\right)^{2}+\sqrt{\langle\alpha\rangle}\left(1+B_{1}^{2}\right)\left(d x-b_{x i} d \zeta^{i}\right)^{2}+\frac{\gamma^{\prime} \sqrt{H}}{\sqrt{\langle\alpha\rangle}} d r^{2} \\
-2 \sqrt{\langle\alpha\rangle} A_{1} B_{1}\left(d x-b_{x i} d \zeta^{i}\right)\left(d y-b_{y i} d \zeta^{j}\right)+\frac{d z^{2}+d_{2}\left|d \chi_{2}\right|^{2}}{\sqrt{\langle\alpha\rangle}}
\end{gathered}
$$

which looks similar to the type I metric that we had in (4.25), as it should. The metric has the usual fibration along the $d x$ and the $d y$ directions and the base is given by $\left(\theta_{1}, \theta_{2}, r, z\right)$ coordinates. The background $B$ field (whose components depend on $r, \theta_{1}$ and $\theta_{2}$ only) that will serve as the torsion and the coupling constant are given by:

$$
\begin{aligned}
H^{\text {het }} \equiv & H=\mathcal{H}_{x z \theta_{1}}^{b} d y \wedge d z \wedge d \theta_{2}-\mathcal{H}_{y z \theta_{2}}^{b} d x \wedge d z \wedge d \theta_{1}+ \\
& +\mathcal{H}_{x z r}^{b} d y \wedge d z \wedge d r-\mathcal{H}_{y z r}^{b} d x \wedge d z \wedge d r \\
g^{\text {het }}= & \frac{1}{\sqrt{\langle\alpha\rangle}} .
\end{aligned}
$$

Along with the solution to the DUY equation this will specify the complete background.

Let us now use the heterotic superpotential (1.4) to determine the $B^{\text {het }}$. As was discussed in [29], [34], one can minimize the superpotential (1.4) to determine the background torsional equation as:

$$
H=* d J
$$

where $J$ is the fundamental two form. This equation also appeared in 6322. Having a non-Kähler manifold means that it will support torsion $H$. For a complex manifold, this

22 In fact in 63 the torsional equation is $H=e^{2 \phi} * d\left(e^{-2 \phi} J\right)$ for manifolds with $S U(3)$ holonomy. This reduces to the torsional equation that we proposed when the dilaton is a constant which, for our case, is true. 
equation can be re-written as

$$
H=i(\partial-\bar{\partial}) J
$$

which is the familiar way the torsional equation first appeared in [36], [44], [69]. In the analysis to follow we will, however, use (5.4) because it is written in terms of real variables. To proceed further, we first need the vielbeins for our space. They are given by:

$$
\begin{aligned}
e^{1} & =\frac{1}{\sqrt{2}}\langle\alpha\rangle^{\frac{1}{4}}\left(1+A_{1}^{2}\right)^{\frac{1}{2}}\left[\left(d y-b_{y j} d \zeta^{j}\right)+\gamma_{2}\left(d x-b_{x i} d \zeta^{i}\right)\right] \\
e^{2} & =\frac{1}{\sqrt{2}}\langle\alpha\rangle^{\frac{1}{4}}\left(1+A_{1}^{2}\right)^{\frac{1}{2}}\left[\left(d y-b_{y j} d \zeta^{j}\right)+\gamma_{3}\left(d x-b_{x i} d \zeta^{i}\right)\right] \\
e^{3} & =\frac{d_{2}^{\frac{1}{2}}}{\langle\alpha\rangle^{\frac{1}{4}}} d \theta_{1}, \quad e^{4}=\frac{\left|\tau_{2}\right| d_{2}^{\frac{1}{2}}}{\langle\alpha\rangle^{\frac{1}{4}}} d \theta_{2}, \quad e^{5}=\frac{\gamma^{\prime \frac{1}{2}} H^{\frac{1}{4}}}{\langle\alpha\rangle^{\frac{1}{4}}} d r, \quad e^{6}=\frac{d z}{\langle\alpha\rangle^{\frac{1}{4}}}
\end{aligned}
$$

where we have already defined $d_{2}$ and $\tau_{2}$. The other variables $\gamma_{2}$ and $\gamma_{3}$ are given as:

$$
\gamma_{2}=-\frac{1}{1+A_{1}^{2}}\left[A_{1} B_{1} \pm \frac{1}{\sqrt{\langle\alpha\rangle}}\right], \quad \gamma_{3}=-\frac{1}{1+A_{1}^{2}}\left[A_{1} B_{1} \mp \frac{1}{\sqrt{\langle\alpha\rangle}}\right] .
$$

Having the vielbeins, we now need to determine the fundamental form $J$, so that we can evaluate $d J$, the deviation from Kählerity. The fundamental form is given by

$$
J=e^{1} \wedge e^{2}+e^{3} \wedge e^{4}+e^{5} \wedge e^{6}=\left[J_{1}\right]_{b_{x \theta_{1}}=b_{y \theta_{2}}=0}+\left[J_{2}\right]
$$

where $d J_{2}$ measures the actual deviance from Kählerity because $d J_{1}=0$ by definition. Now we can easily evaluate $J_{2}$ from the vielbeins $(5.6)$, it is found to be:

$$
J_{2}=-\left[b_{x i} d y \wedge d \zeta^{i}-b_{y j} d x \wedge d \zeta^{j}+b_{x i} b_{y j} d \zeta^{i} \wedge d \zeta^{j}\right]
$$

Using (5.4) we get the following results for the three form $H$ :

$$
\begin{aligned}
& H_{r x z}=\partial_{[r} B_{x z]}^{\text {het }}=\eta_{1} \partial_{\left[\theta_{2}\right.} b_{\left.x \theta_{1}\right]}, \quad H_{\theta_{2} x z}=\partial_{\left[\theta_{2}\right.} B_{x z]}^{\text {het }}=\eta_{2} \partial_{[r} b_{\left.x \theta_{1}\right]} \\
& \left.H_{\theta_{1} y z}=\partial_{\left[\theta_{1}\right.} B_{y z]}^{\text {het }}=\eta_{3} \partial_{[r} b_{\left.y \theta_{2}\right]}, \quad H_{r y z}=\partial_{[r} B_{y z]}^{\text {het }}=\eta_{4} \partial_{\left[\theta_{1}\right.} b_{y \theta_{2}}\right]
\end{aligned}
$$

along with another relation:

$$
H_{x y z}=\eta_{5} \partial_{[x} B_{y z]}^{\mathrm{het}}=0
$$

which vanishes because we have taken the $B^{\text {het }}$ to be functions of $\left(\theta_{1}, \theta_{2}, r\right)$ and independent of $(x, y, z)$. The variables $\eta_{i}$ can be evaluated from the epsilon tensor and Hodge star. 
To compare (5.10) with (4.42), observe that

$$
H_{x z\left(\theta_{1}, r\right)}^{\text {het }}=-\mathcal{H}_{y z\left(\theta_{2}, r\right)}^{b}, \quad H_{y z\left(\theta_{2}, r\right)}^{\text {het }}=\mathcal{H}_{x z\left(\theta_{1}, r\right)}^{b}
$$

which is basically (5.3). Plugging this in (5.10) we get:

$$
\begin{array}{ll}
\mathcal{H}_{r y z}^{b}=-\eta_{1} \partial_{\left[\theta_{2}\right.} b_{\left.x \theta_{1}\right]}, & \mathcal{H}_{\theta_{2} y z}^{b}=-\eta_{2} \partial_{[r} b_{\left.x \theta_{1}\right]} \\
\mathcal{H}_{\theta_{1} x z}^{b}=\eta_{3} \partial_{[r} b_{\left.y \theta_{2}\right]}, & \mathcal{H}_{r x z}^{b}=\eta_{4} \partial_{\left[\theta_{1}\right.} b_{\left.y \theta_{2}\right]} .
\end{array}
$$

Comparing (5.13) with (4.42), we see that if we impose the following conjectured conditions on $\alpha_{i}$ and $\eta_{i}$

$$
\alpha_{1}=-\eta_{1}, \quad \alpha_{2}=-\eta_{2}, \quad \alpha_{3}=\eta_{3}, \quad \alpha_{4}=\eta_{4}
$$

then we obtain perfect equality! The reason why we called this conjectured condition is because $\alpha_{i}$ are constants as we had used the limit (2.6) to go to the metric at the orientifold point. Away from this limit, $\alpha_{i}$ do not need to be constants. In that case they have to be determined using the $B$ fields, especially $b_{x i}$ and $b_{y j}$. Therefore, we expect $\alpha_{i}$ to be functions of $b_{x i}$ and $b_{y j}$. On the other hand $\eta_{i}$ are in fact functions of $b_{x i}$ and $b_{y j}$, as can be easily seen by working out the Hodge star. This is exactly what the equality (5.14) implies here.

\subsection{Background after geometric transition}

After the geometric transition in type IIB theory the dual metric from the type I picture can be easily brought in the heterotic framework via S-duality in the same manner as before. The metric is now:

$$
\begin{gathered}
d s^{2}=\frac{1}{\left(h_{2}+a_{2}^{2} h_{1}\right) \sqrt{C^{\prime} D^{\prime}}}\left(d y-b_{y j} d \zeta^{j}\right)^{2}+\frac{1}{\left(h_{4}+a_{1}^{2} h_{1}\right) \sqrt{C^{\prime} D^{\prime}}}\left(d x-b_{x i} d \zeta^{i}\right)^{2} \\
+\frac{h_{1}}{\sqrt{C^{\prime} D^{\prime}}} d z^{2}+\frac{h_{3} h_{4}}{\sqrt{C^{\prime} D^{\prime}}}\left|d \chi_{2}\right|^{2}+\frac{\gamma^{\prime} \sqrt{H}}{\sqrt{C^{\prime} D^{\prime}}} d r^{2} .
\end{gathered}
$$

which is again similar to the type I picture developed earlier, i.e. (4.52). The torsion and the coupling are

$$
\begin{aligned}
H_{\text {het }} \equiv & \tilde{H}=\tilde{\mathcal{H}}_{x z \theta_{1}}^{b} d y \wedge d z \wedge d \theta_{2}-\tilde{\mathcal{H}}_{y z \theta_{2}}^{b} d x \wedge d z \wedge d \theta_{1}+ \\
& +\tilde{\mathcal{H}}_{x z r}^{b} d y \wedge d z \wedge d r-\tilde{\mathcal{H}}_{y z r}^{b} d x \wedge d z \wedge d r \\
g^{\text {het }} & =\frac{1}{\sqrt{C^{\prime} D^{\prime}}}
\end{aligned}
$$


which, as before, along with the solution to the DUY equation will specify the complete background.

To verify the torsional equation we can follow the same steps, with the $H$ 's replaced by $\tilde{H}$. It is easy to see that torsional equations similar to (5.13) are satisfied here, too. However, for completeness we give the vielbeins for this case:

$$
\begin{aligned}
& e^{1}=\left(h_{2}+a_{2}^{2} h_{1}\right)^{-\frac{1}{2}}\left(C^{\prime} D^{\prime}\right)^{-\frac{1}{4}}\left(d y-b_{y j} d \zeta^{j}\right), \quad e^{2}=\left(h_{4}+a_{1}^{2} h_{1}\right)^{-\frac{1}{2}}\left(C^{\prime} D^{\prime}\right)^{-\frac{1}{4}}\left(d x-b_{x i} d \zeta^{i}\right) \\
& e^{3}=\frac{1}{\sqrt{2}}\left(h_{3} h_{4}\right)^{\frac{1}{2}}\left(C^{\prime} D^{\prime}\right)^{-\frac{1}{4}}\left(d \theta_{1}+\gamma_{4} d \theta_{2}\right), \quad e^{4}=\frac{1}{\sqrt{2}}\left(h_{3} h_{4}\right)^{\frac{1}{2}}\left(C^{\prime} D^{\prime}\right)^{-\frac{1}{4}}\left(d \theta_{1}+\gamma_{5} d \theta_{2}\right) \\
& e^{5}=\gamma^{\frac{1}{2}} H^{\frac{1}{4}}\left(C^{\prime} D^{\prime}\right)^{-\frac{1}{4}} d r, \quad e^{6}=h_{1}^{\frac{1}{2}}\left(C^{\prime} D^{\prime}\right)^{-\frac{1}{4}} d z
\end{aligned}
$$

where we now see that the vielbeins $e^{1}$ and $e^{2}$ are simple because $\operatorname{Re} \tau_{1}=0$, whereas the vielbeins $e^{3}$ and $e^{4}$ contain mixed components because $\operatorname{Re} \tau_{2} \neq 0$. For (5.6) this was exactly the opposite. It is intriguing to see that, for generic $c$, before geometric transition the heterotic dual manifold has $\operatorname{Re} \tau_{1} \neq 0$, Re $\tau_{2}=0$ and after geometric transition the heterotic dual manifold has $\operatorname{Re} \tau_{1}=0, \operatorname{Re} \tau_{2} \neq 0$. The variables $\gamma_{4}$ and $\gamma_{5}$ appearing in (5.17) are defined as:

$$
\gamma_{4}=\operatorname{Re} \tau_{2} \pm \operatorname{Im} \tau_{2}, \quad \gamma_{5}=\operatorname{Re} \tau_{2} \mp \operatorname{Im} \tau_{2}
$$

where $\tau_{2}$ was defined in (4.48).

At this point let us pause a little to ask what the two backgrounds derived above mean. It is clear that the dual background before geometric transition is related to NS5 branes wrapped on two cycles of the non-Kähler manifold (5.2). Following the duality chain, this comes from the D5 wrapped on a two cycle of a resolved conifold (which survives the orientifold operation). After the geometric transition we get a background that has only fluxes but no branes. The question here is whether we can shrink the two cycle on which we have wrapped NS5 branes and get another background with fluxes. At a more fundamental level, can the closed string background with fluxes compute anything of the world volume dynamics on the wrapped NS5 branes? We do not have any answer to this question yet. We will discuss a bit more on this in the next section from the superpotential point of view.

A similar question can also be raised for the type I setting. The dynamics on the wrapped $D 5$ branes are known. It is the same theory as for the type IIB case. Whether the closed string background that we gave earlier is a dual background for the D5 brane theory can only be clear after some concrete analysis is performed. 


\section{Field Theory and Geometric Transitions in Type I and Heterotic String}

We now consider the primary region of interest in the above discussion, i.e. the orientifold point. The field theory on the D5 brane will change from the usual one to a theory describing D5 branes wrapped on a $P^{1}$ cycle of a resolved conifold. In the language of type I, the paper [70] discussed the field theory on $N$ D5 branes which appear as $N$ instantons. When all the $N$ D 5 branes coincide, the field theory gains an unbroken $S p(N)$ symmetry. The T-dual type IIB picture will replace the $N$ D5 branes with another set of $N$ D 5 branes and there will exist an unbroken $\operatorname{Sp}(N)$ symmetry on the D 5 branes.

The field theory on the $N$ wrapped D5 branes is similar to the one on the probe D3 branes considered in type IIB in [71]. The difference is that the wrapped D5 branes are fixed

on the $P^{1}$ cycle so that the antisymmetric hypermultiplet (which comes from projecting an $\mathcal{N}=2$ adjoint field) is not part of the theory. We only have an $\mathcal{N}=2, S p(N)$ theory with four flavors of matter field in the fundamental representation (given by the four D7 branes which touch the D5 brane).

What is the superpotential of the theory? This can be obtained from the field configuration present after the transition. It involves $H_{R R}$ instead of D5 branes and $H_{N S}$. Besides, the orientifold O7 plane and the D7 branes survive the transition. They will be grouped identically as in the configuration before the transition. Therefore one is still at the orientifold point where the axion and dilaton do not vary. The well known superpotential

$$
W_{I I B}=\int\left(H_{R R}+\tau H_{N S}\right) \wedge \Omega
$$

still appears. Here $\Omega$ is the usual holomorphic $(3,0)$ form of the base discussed earlier. But there is another contribution to the superpotential which comes from the D7 branes and this can be calculated using arguments similar to the ones in [72. The contribution of the D7 branes appears as a Chern-Simons term analog to the holomorphic ChernSimons computation for D5 branes wrapped on holomorphic cycles. Even though it is now more difficult to identify the four-cycles around which the D7 branes are wrapped, the computation of the Chern-Simons action as a function of the complex structure of the manifold would proceed similarly to [72].

We now study the transformation of the type IIB superpotential under two Tdualities. In the type I theory we get several contributions to the superpotential. The first one appears as $\int d B_{R R} \wedge \Omega$, which originates from the similar term appearing in the 
type IIB theory. The second one comes as $d J \wedge \Omega$, [29], [34] and is due to the fact that the type I theory is compactified on a non-Kähler manifold which has a non-zero $d J$.

The third and the most intriguing one appears as a holomorphic Chern-Simons functional

$$
W_{C S}^{A}=\int \operatorname{tr}\left(A \wedge d A+\frac{2}{3} A \wedge A \wedge A\right) \wedge \Omega,
$$

where $A$ is the $\mathrm{SO}(32)$ gauge field. One would naively think that this term appears as an extra term compared to the type IIB theory but, in view of the above discussion, it is not. This contribution actually arises rather naturally because of the extra Chern-Simons contribution coming from the field theory on the D7 branes, which survive the transition 23. The two Chern-Simons terms can, and should, be mapped into each other.

The most generic superpotential for the type I theory is then [29], 34]

$$
W_{I}=\int\left(d B_{R R}+i d J\right) \wedge \Omega+W_{C S}^{A}+W_{C S}^{\omega},
$$

where $W_{C S}^{A}$ and $W_{C S}^{\omega}$ are the gauge and the gravitational Chern-Simons terms, respectively. This will describe the low-energy effective theory on the small instanton D5 branes of the type I superstring.

Performing an S-duality to go from type I string to the heterotic string, the D5 branes become NS5 branes, the field $H_{R R}$ becomes $H_{N S} \equiv H$ and the compactification manifold remains non-Kähler. The superpotential therefore is [29], [34]

$$
W_{\text {het }}=\int(H+i d J) \wedge \Omega,
$$

where $H$ is the usual three-form of the heterotic theory satisfying $d H=\operatorname{tr} R \wedge R-\frac{1}{30} \operatorname{tr} F \wedge$ $F$. Furthermore, the D9 branes and $O 9$ planes have disappeared and the flavor degrees of freedom now live on the $S O(32)$ vector bundle24.

What about the possibility of having a gluino condensate in the heterotic string as described in [73]? The combination of fluxes and a gluino condensate has been considered in [35] 42] where it was argued that the superpotential (6.4) becomes

$$
W_{h e t}=\int(H+i d J+\Sigma) \wedge \Omega,
$$

23 For a derivation of this see 32].

24 In the examples that we studied in this paper the gauge group is broken to a smaller subgroup by Wilson lines. These Wilson lines map to the distances between the stack of $D 7$ branes in the original type IIB picture. 
where $\Sigma$ is the expectation value for $\operatorname{tr}\left(\bar{\chi} \Gamma_{m n p} \chi\right), \chi$ being the gluino. For compact Calabi-Yau, considered in [42], this typically fixes both the radius and the dilaton nonperturbatively25.

The gluino condensate in equation (6.5) is different from the gluino condensate for the field theory living on the NS5 branes. The NS5 brane is the S-dual of the type I D5 brane and the field theory on the NS5 brane appears in the decoupling limit as discussed in [3]. The gauge coupling constant for the theory on the NS5 brane is inverse proportional to the volume of the $P^{1}$ on which the NS5 is wrapped on.

The bulk gauge coupling constant is given by $e^{\phi}$, where $\phi$ is the four dimensional dilaton given by [42]

$$
\phi=\frac{\Phi}{2}-6 \sigma
$$

where $\Phi$ is the ten dimensional dilaton and $\sigma$ is the volume modulus. The volume scalar $\rho$ is [42]

$$
\rho=\frac{\Phi}{2}+2 \sigma
$$

Now, because the internal manifold is non-compact, $\sigma$ is infinite so $\phi$ goes to minus infinity and $\rho$ goes to infinity. Because the gluino condensate is the exponential of a combination of $-e^{\rho}$ and $-e^{-\phi}$, in this limit it reduces to zero. This is indeed the expected result for a non-compact manifold. This is because the initial type IIB picture contains non-compact D7 branes and the gluino condensate on the D7 branes is then zero.

\subsection{Geometric Transitions}

We now would like to discuss the geometric transitions which type I strings and heterotic strings should inherit from the type IIB theory after T-dualities and S-duality.

In type IIB theory geometric transitions, the superpotential is a holomorphic function which is computed using topological strings on both the open string and the closed string side. For the case of type IIA theory on the conifold, the results have been matched in [2], where the non-Kählerity and non-complexity on the closed string side was first observed. The non-zero $d \Omega$ on the closed string side controls the field theory bare coupling constant.

25 There is an interesting simplification when we go to the compact non-Kähler case. Due to the torsional equation (5.4) and the Bianchi identity of $d H^{\text {het }}$ the scaling freedom of the fundamental two form $J$ is broken. This means that the radius of the manifold is fixed at tree level! The fact that there exists a potential for the radial modulus which has a minimum was shown in [29]. Thus, this leaves us to fix only the dilaton non-perturbatively. 
The question now is whether the same topological string computations can be performed for our case. In view of the original introduction of topological strings [74], these objects are obtained after twisting two dimensional $(2,2)$ theories. Progress has also been made in defining topological strings for heterotic string theory [40]. Even though the left-right symmetry of the worldsheet is absent in the heterotic case, the F-term of the $\mathcal{N}=1$ supersymmetric theory is related to topological quantities. The topological partition function is related in this case to $\mathrm{F}$-terms like $W^{2 g}$, where $W$ is now the $\mathcal{N}=1$ gauge multiplet.

It would be very interesting to go further in this direction. This could provide us with a recipe for computing the superpotential by using the twisted topological $(0,2)$ theory. One should remember that there is an extra ingredient due to the departure of the metric from being complex and Kähler. In terms of topological strings, there should be an extra change similar to the one appearing in the recent proposal of [75] for the $(2,2)$ theories. A similar change due to the presence of torsion would be naturally expressed in terms of some generalized complex geometry. In what concerns the actual computation of the superpotential in terms of the topological string, it should be shown that the new open topological strings contribute in a similar way to the ones of the A-model and B-model. Once such a picture becomes clear, then it will be possible to see whether we can really observe a geometric transition in heterotic or type I theory. The wrapped D5 branes on a two-cycle of the non-Kähler manifold in type I theory should be replaced by the other background that we gave which had fluxes supported on another non-Kähler manifold. The dynamics on the wrapped $D 5$ should also be obtained from this background26.

Another interesting future direction is to use our results as a tool for understanding mirror symmetry for $(0,2)$ theories [37 38] [39]. As we have the metrics and we know how to transform between mirror symmetric solutions in terms of $\mathrm{T}$-dualities, one could try to describe the mirror of the heterotic theory.

\section{Acknowledgments}

Its our pleasure to thank Gianguido Dall' Agata, Shamit Kachru, Amir-Kian KashaniPoor, Dieter Luest, and Mohammed M. Sheikh-Jabbari for many interesting discussions and useful correspondences. The work of S.A. is supported by US DOE under grant DEAC03-76SF00515. The work of K.B. is supported by NSF grant PHY-0244722, an Alfred

26 We are assuming here that the theory on the wrapped $D 5$ branes is decoupled theory from the bulk dynamics. Otherwise we have to deal with a gauge theory coupled with gravity. 
Sloan Fellowship and the University of Utah. The work of M.B. is supported by NSF grant PHY-01-5-23911 and an Alfred Sloan Fellowship. The work of K.D. is supported in part by a Lucile and David Packard Foundation Fellowship 2000-13856. A.K. would like to acknowledge support from the University of Maryland. R.T. is supported by DOE Contract DE-AC03-76SF0098 and NSF grant PHY-0098840. 


\section{References}

[1] I. R. Klebanov and M. J. Strassler, "Supergravity and a confining gauge theory: Duality cascades and $\chi$ - SB-resolution of naked singularities," JHEP 0008, 052 (2000), hep-th/0007191.

[2] C. Vafa, "Superstrings and topological strings at large N," J. Math. Phys. 42, 2798 (2001), hep-th/0008142.

[3] J. M. Maldacena and C. Nunez, "Towards the large N limit of pure $\mathrm{N}=1$ super Yang Mills," Phys. Rev. Lett. 86, 588 (2001), hep-th/0008001.

[4] R. Gopakumar and C. Vafa, "On the gauge theory/geometry correspondence," Adv. Theor. Math. Phys. 3, 1415 (1999),hep-th/9811131.

[5] F. Cachazo, K. A. Intriligator and C. Vafa, "A large N duality via a geometric transition," Nucl. Phys. B 603, 3 (2001), hep-th/0103067.

[6] J. D. Edelstein, K. Oh and R. Tatar, "Orientifold, geometric transition and large N duality for SO/Sp gauge theories," JHEP 0105, 009 (2001), hep-th/0104037.

[7] F. Cachazo, S. Katz and C. Vafa, "Geometric transitions and N = 1 quiver theories," hep-th/0108120.

[8] F. Cachazo, B. Fiol, K. A. Intriligator, S. Katz and C. Vafa, "A geometric unification of dualities," Nucl. Phys. B 628, 3 (2002), hep-th/0110028.

[9] K. h. Oh and R. Tatar, "Duality and confinement in $\mathrm{N}=1$ supersymmetric theories from geometric transitions," Adv. Theor. Math. Phys. 6, 141 (2003), hep-th/0112040.

[10] R. Dijkgraaf and C. Vafa, "A perturbative window into non-perturbative physics," hep-th/0208048.

[11] R. Dijkgraaf and C. Vafa, "Matrix models, topological strings, and supersymmetric gauge theories," Nucl. Phys. B 644, 3 (2002), hep-th/0206255.

[12] S. Gukov, C. Vafa and E. Witten, "CFT's from Calabi-Yau four-folds," Nucl. Phys. B 584, 69 (2000) [Erratum-ibid. B 608, 477 (2001), hep-th/9906070.

[13] S. Gurrieri, J. Louis, A. Micu and D. Waldram, "Mirror symmetry in generalized Calabi-Yau compactifications," Nucl. Phys. B 654, 61 (2003), hep-th/0211102.

[14] S. Chiossi, S. Salamon, "The intrinsic torsion of $S U(3)$ and $G_{2}$ structures," Proc. conf. Differential Geometry Valencia 2001.

[15] M. Becker, K. Dasgupta, A. Knauf and R. Tatar, "Geometric transitions, flops and non-Kaehler manifolds. I," hep-th/0403288.

[16] P. Kaste, R. Minasian, M. Petrini and A. Tomasiello, "Nontrivial RR two-form field strength and SU(3)-structure," Fortsch. Phys. 51, 764 (2003), hep-th/0301063 ; S. Fidanza, R. Minasian,A. Tomasiello,"Mirror Symmetric SU(3) Structure Manifolds with NS fluxes", hep-th/0311122; M. Grana, R. Minasian, M. Petrini and A. Tomasiello, "Supersymmetric backgrounds from generalized Calabi-Yau manifolds," hep-th/0406137. 
[17] S. Gurrieri and A. Micu, "Type IIB theory on half-flat manifolds," Class. Quant. Grav. 20, 2181 (2003), hep-th/0212278.

[18] G. Dall'Agata and N. Prezas, "N=1 geometries for M-theory and type IIA strings with fluxes," hep-th/0311146; G. Dall'Agata, "On Supersymmetric Solutions of Type IIB Supergravity with General Fluxes", hep-th/0403220.

[19] K. Behrndt and M. Cvetic,K. Behrndt and M. Cvetic, "General N = 1 supersymmetric flux vacua of (massive) type IIA string theory," hep-th/0403049; "General N = 1 supersymmetric fluxes in massive type IIA string theory," hep-th/0407263.

[20] L. A. Pando Zayas and A. A. Tseytlin, "3-branes on resolved conifold," JHEP 0011, 028 (2000), hep-th/0010088.

[21] A. Strominger, S. T. Yau and E. Zaslow, "Mirror symmetry is T-duality," Nucl. Phys. B 479, 243 (1996), hep-th/9606040.

[22] K. Dasgupta, K. Oh and R. Tatar, "Geometric transition, large N dualities and MQCD dynamics," Nucl. Phys. B 610, 331 (2001), hep-th/0105066; "Open/closed string dualities and Seiberg duality from geometric transitions in M-theory," JHEP 0208, 026 (2002), hep-th/0106040.

[23] K. Dasgupta, K. h. Oh, J. Park and R. Tatar, "Geometric transition versus cascading solution," JHEP 0201, 031 (2002), hep-th/0110050.

[24] I. Bena, R. Roiban and R. Tatar, "Baryons, boundaries and matrix models," Nucl. Phys. B 679, 168 (2004), hep-th/0211271; I. Bena, H. Murayama, R. Roiban and R. Tatar, "Matrix model description of baryonic deformations," JHEP 0305, 049 (2003), hep-th/0303115.

[25] R. Roiban, R. Tatar and J. Walcher, "Massless flavor in geometry and matrix models," Nucl. Phys. B 665, 211 (2003), hep-th/0301217.

[26] K. Landsteiner, C. I. Lazaroiu and R. Tatar, "(Anti)symmetric matter and superpotentials from IIB orientifolds," JHEP 0311, 044 (2003), hep-th/0306236; "Chiral field theories, Konishi anomalies and matrix models," JHEP 0402, 044 (2004), hep-th/0307182; "Chiral field theories from conifolds," JHEP 0311, 057 (2003), hepth/0310052; "Puzzles for matrix models of chiral field theories," Fortsch. Phys. 52, 590 (2004), hep-th/0311103.

[27] J. F. G. Cascales and A. M. Uranga, "Branes on generalized calibrated submanifolds," hep-th/0407132.

[28] A. Sen, "F-theory and Orientifolds," Nucl. Phys. B 475, 562 (1996), hep-th/9605150; 'Orientifold limit of F-theory vacua," Phys. Rev. D 55, 7345 (1997), hep-th/9702165; "Orientifold limit of F-theory vacua," Nucl. Phys. Proc. Suppl. 68, 92 (1998) [Nucl. Phys. Proc. Suppl. 67, 81 (1998)], hep-th/9709159.

[29] K. Becker, M. Becker, K. Dasgupta and S. Prokushkin, "Properties of heterotic vacua from superpotentials," Nucl. Phys. B 666, 144 (2003), hep-th/0304001. 
[30] K. Dasgupta, G. Rajesh and S. Sethi, "M theory, orientifolds and G-flux," JHEP 9908, 023 (1999), hep-th/9908088.

[31] K. Becker and K. Dasgupta, "Heterotic strings with torsion," JHEP 0211, 006 (2002), hep-th/0209077.

[32] K. Becker, M. Becker, K. Dasgupta and P. S. Green, "Compactifications of heterotic theory on non-Kähler complex manifolds. I," JHEP 0304, 007 (2003), hep-th/0301161.

[33] K. Becker, M. Becker, P. S. Green, K. Dasgupta and E. Sharpe, "Compactifications of heterotic strings on non-Kähler complex manifolds. II," Nucl. Phys. B 678, 19 (2004), hep-th/0310058.

[34] G. L. Cardoso, G. Curio, G. Dall'Agata and D. Lust, "BPS action and superpotential for heterotic string compactifications with fluxes," JHEP 0310, 004 (2003), hepth/0306088.

[35] G. L. Cardoso, G. Curio, G. Dall'Agata and D. Lust, "Heterotic string theory on non-Kähler manifolds with H-flux and gaugino condensate," hep-th/0310021.

[36] A. Strominger, "Superstrings with torsion," Nucl. Phys. B 274, 253 (1986).

[37] R. Blumenhagen, R. Schimmrigk and A. Wisskirchen, "(0,2) mirror symmetry," Nucl. Phys. B 486, 598 (1997), hep-th/9609167.

[38] A. Adams, A. Basu and S. Sethi, "(0,2) duality," Adv. Theor. Math. Phys. 7, 865 (2004), hep-th/0309226.

[39] S. Katz and E. Sharpe, "Notes on certain (0,2) correlation functions," hep-th/0406226.

[40] I. Antoniadis, E. Gava, K. S. Narain and T. R. Taylor, "Topological Amplitudes in Heterotic Superstring Theory," Nucl. Phys. B 476, 133 (1996), hep-th/9604077.

[41] S. Kachru, R. Kallosh, A. Linde and S. P. Trivedi, "De Sitter vacua in string theory," Phys. Rev. D 68, 046005 (2003), hep-th/0301240.

[42] S. Gukov, S. Kachru, X. Liu and L. McAllister, "Heterotic moduli stabilization with fractional Chern-Simons invariants," Phys. Rev. D 69, 086008 (2004), hep-th/0310159.

[43] M. Becker, G. Curio and A. Krause, "De Sitter vacua from heterotic M Theory," Nucl. Phys. B 693, 223 (2004), hep-th/0403027.

[44] C. M. Hull, "Superstring Compactifications With Torsion And Space-Time Supersymmetry," Print-86-0251 (CAMBRIDGE), Published in Turin Superunif.1985:347; C. M. Hull and E. Witten, "Supersymmetric Sigma Models And The Heterotic String," Phys. Lett. B 160, 398 (1985).

[45] S. Gurrieri, A. Lukas, A. Micu, "Heterotic on Half-flat" hep-th/0408121.

[46] Y. Imamura, "Born-Infeld action and Chern-Simons term from Kaluza-Klein monopole in M-theory," Phys. Lett. B 414, 242 (1997), hep-th/9706144; A. Sen, "Dynamics of multiple Kaluza-Klein monopoles in M and string theory," Adv. Theor. Math. Phys. 1, 115 (1998), hep-th/9707042; "A note on enhanced gauge symmetries in M- and string theory," JHEP 9709, 001 (1997), hep-th/9707123. 
[47] K. Dasgupta, G. Rajesh, D. Robbins and S. Sethi, "Time-dependent warping, fluxes, and NCYM," JHEP 0303, 041 (2003), hep-th/0302049; K. Dasgupta and M. Shmakova, "On branes and oriented B-fields," Nucl. Phys. B 675, 205 (2003), hep-th/0306030.

[48] K. Becker and M. Becker, "M-Theory on eight-manifolds," Nucl. Phys. B 477, 155 (1996), hep-th/9605053.

[49] S. B. Giddings, S. Kachru and J. Polchinski, "Hierarchies from fluxes in string compactifications," Phys. Rev. D 66, 106006 (2002), hep-th/0105097.

[50] A. M. Uranga, "Brane configurations for branes at conifolds," JHEP 9901, 022 (1999), hep-th/9811004.

[51] K. Dasgupta and S. Mukhi, "Brane constructions, conifolds and M-theory," Nucl. Phys. B 551, 204 (1999), hep-th/9811139.

[52] R. de Mello Koch, K. Oh and R. Tatar, "Moduli space for conifolds as intersection of orthogonal D6 branes," Nucl. Phys. B 555, 457 (1999), hep-th/9812097.

[53] K. Dasgupta and S. Mukhi, "F-theory at constant coupling," Phys. Lett. B 385, 125 (1996), hep-th/9606044.

[54] T. Banks, M. R. Douglas and N. Seiberg, "Probing F-theory with branes," Phys. Lett. B 387, 278 (1996), hep-th/9605199.

[55] N. Seiberg and E. Witten, "Electric - magnetic duality, monopole condensation, and confinement in N=2 supersymmetric Yang-Mills theory," Nucl. Phys. B 426, 19 (1994) [Erratum-ibid. B 430, 485 (1994)], hep-th/9407087; "Monopoles, duality and chiral symmetry breaking in N=2 supersymmetric QCD," Nucl. Phys. B 431, 484 (1994), hep-th/9408099

[56] M. Grana and J. Polchinski, "Gauge / gravity duals with holomorphic dilaton," Phys. Rev. D 65, 126005 (2002), hep-th/0106014.

[57] O. Aharony, A. Fayyazuddin and J. M. Maldacena, "The large N limit of N = 2,1 field theories from three-branes in F-theory," JHEP 9807, 013 (1998), hep-th/9806159.

[58] K. Dasgupta, D. P. Jatkar and S. Mukhi, "Gravitational couplings and Z(2) orientifolds," Nucl. Phys. B 523, 465 (1998), hep-th/9707224.

[59] S. Sethi, C. Vafa and E. Witten, "Constraints on low-dimensional string compactifications," Nucl. Phys. B 480, 213 (1996), hep-th/9606122; K. Dasgupta and S. Mukhi, "A note on low-dimensional string compactifications," Phys. Lett. B 398, 285 (1997), hep-th/9612188.

[60] A. Karch, D. Lust and D. J. Smith, "Equivalence of geometric engineering and HananyWitten via fractional branes," Nucl. Phys. B 533, 348 (1998), hep-th/9803232.

[61] B. A. Burrington, J. T. Liu, L. A. Pando Zayas and D. Vaman, "Holographic duals of flavored N = 1 super Yang-Mills: Beyond the probe approximation," hep-th/0406207.

[62] S. Kachru, M. B. Schulz and S. Trivedi, "Moduli stabilization from fluxes in a simple IIB orientifold," JHEP 0310, 007 (2003), hep-th/0201028; S. Kachru, M. B. Schulz, 
P. K. Tripathy and S. P. Trivedi, "New supersymmetric string compactifications," JHEP 0303, 061 (2003), hep-th/0211182; M. B. Schulz, "Superstring orientifolds with torsion: O5 orientifolds of torus fibrations and their massless spectra," hep-th/0406001

[63] J. P. Gauntlett, D. Martelli and D. Waldram, "Superstrings with intrinsic torsion," Phys. Rev. D 69, 086002 (2004), hep-th/0302158.

[64] S. S. Gubser, "Supersymmetry and F-theory realization of the deformed conifold with three-form flux," hep-th/0010010; A. Giveon, A. Kehagias and H. Partouche, "Geometric transitions, brane dynamics and gauge theories," JHEP 0112, 021 (2001), hep-th/0110115.

[65] E. Goldstein and S. Prokushkin, "Geometric model for complex non-Kähler manifolds with SU(3) structure," hep-th/0212307.

[66] G. L. Cardoso, G. Curio, G. Dall'Agata, D. Lust, P. Manousselis and G. Zoupanos, "Non-Kaehler string backgrounds and their five torsion classes," Nucl. Phys. B 652, 5 (2003), hep-th/0211118.

[67] P. Candelas, G. T. Horowitz, A. Strominger and E. Witten, "Vacuum Configurations For Superstrings," Nucl. Phys. B 258, 46 (1985).

[68] C. M. Hull, "Sigma Model Beta Functions And String Compactifications," Nucl. Phys. B 267, 266 (1986).

[69] B. de Wit, D. J. Smit and N. D. Hari Dass, "Residual Supersymmetry Of Compactified D = 10 Supergravity," Nucl. Phys. B 283, 165 (1987).

[70] E. Witten, "Small Instantons in String Theory," Nucl. Phys. B 460, 541 (1996), hepth/9511030.

[71] M. R. Douglas, D. A. Lowe and J. H. Schwarz, "Probing F-theory with multiple branes," Phys. Lett. B 394, 297 (1997), hep-th/9612062

[72] R. Roiban, R. Tatar and J. Walcher, "Massless flavor in geometry and matrix models," Nucl. Phys. B 665, 211 (2003), hep-th/0301217.

[73] M. Dine, R. Rohm, N. Seiberg and E. Witten, "Gluino Condensation In Superstring Models," Phys. Lett. B 156, 55 (1985).

[74] E. Witten, "Topological Sigma Models," Commun. Math. Phys. 118, 411 (1988).

[75] A. Kapustin and Y. Li, "Topological sigma-models with H-flux and twisted generalized complex manifolds," hep-th/0407249 